\title{
Negative Index Materials: Some Mathematical Perspectives
}

\author{
Hoai-Minh Nguyen ${ }^{1}$
}

Received: 20 December 2017 / Revised: 2 March 2018 / Accepted: 8 March 2018

(C) Institute of Mathematics, Vietnam Academy of Science and Technology (VAST) and Springer Nature

Singapore Pte Ltd. 2018

\begin{abstract}
Negative index materials are artificial structures whose refractive index has a negative value over some frequency range. These materials were postulated and investigated theoretically by Veselago in 1964 and were confirmed experimentally by Shelby, Smith, and Schultz in 2001. New fabrication techniques now allow for the construction of negative index materials at scales that are interesting for applications, which has made them a very active topic of investigation. In this paper, we report various mathematical results on the properties of negative index materials and their applications. The topics discussed herein include superlensing using complementary media, cloaking using complementary media, cloaking an object via anomalous localized resonance, and the well-posedness and the finite speed propagation in media consisting of dispersive metamaterials. Some of the results have been refined and have simpler proofs than the original ones.
\end{abstract}

Keywords Superlensing · Cloaking · Finite speed propagation · Complementary media · Negative index metamaterials.

Mathematics Subject Classification (2010) 35B34 · 35B35 · 35J05 · 35Q60.

\section{Introduction}

Negative index materials (NIMs) are artificial structures whose refractive index has a negative value over some frequency range. These materials were postulated and investigated theoretically by Veselago in 1964 [45] and their existence was confirmed experimentally by Shelby, Smith, and Schultz in 2001 [44]. New fabrication techniques now allow for the construction of NIMs at scales that are interesting for applications, which has made them

Lecture at the Annual Meeting 2017 of the Vietnam Institute for Advanced Study in Mathematics

Hoai-Minh Nguyen

hoai-minh.nguyen@epfl.ch

1 Department of Mathematics, EPFL SB CAMA, Station 8, 1015 Lausanne, Switzerland 
a very active topic of investigation. NIMs have attracted a lot of attention from the scientific community, not only because of potentially interesting applications, but also because of challenges involved in understanding their peculiar properties due to the sign-changing coefficients in the equations modeling the phenomena.

Concerning the electromagnetic wave, wave phenomena in the time harmonic regime are modeled by Maxwell equations

$$
\left\{\begin{array}{cc}
\nabla \times E=i k \mu H & \text { in } \mathbb{R}^{3}, \\
\nabla \times H=-i k \epsilon E+j & \text { in } \mathbb{R}^{3} .
\end{array}\right.
$$

Here $\epsilon$ and $\mu$ are $3 \times 3$ matrix-valued functions corresponding to the permittivity and permeability of the medium, respectively, $j$ is the density of charge, $k>0$ is the frequency, and $i$ is the pure imaginary complex number $\left(i^{2}=-1\right)$. NIMs fall into the region in which both $\epsilon$ and $\mu$ are negative (in the matrix sense); for a standard material, both $\epsilon$ and $\mu$ are positive. Concerning the acoustic wave, phenomena in the time harmonic regime are modeled by the Helmholtz equation

$$
\operatorname{div}(\mathbf{A} \nabla u)+k^{2} \boldsymbol{\Sigma} u=f \text { in } \mathbb{R}^{d}
$$

with $d=2,3$ where $\mathbf{A}$ is a $d \times d$ matrix-valued function and $\boldsymbol{\Sigma}$ is a function that describes the properties of the medium. For NIMs, $\mathbf{A}$ and $\boldsymbol{\Sigma}$ are negative; for a standard material, both $\mathbf{A}$ and $\boldsymbol{\Sigma}$ are positive. In the acoustic quasistatic regime $k=0$, the medium is therefore characterized by the matrix $\mathbf{A}$. This regime will be discussed in detail in this paper to illustrate the phenomena and mathematical ideas used to investigate NIMs with an exception in Section 5 where only the electromagnetic setting is considered. Facts related to the finite frequency regime and the electromagnetic setting are also mentioned.

To correctly investigate these equations, one adds some loss (friction or damping effects) into the region of NIMs and then studies these equations as the loss goes to 0. Mathematically, the study of media consisting of NIMs faces two difficulties. First, the equations describing the wave phenomena have sign-changing coefficients; hence, the ellipticity and the compactness are lost in general. Second, a localized resonance, i.e., the field (the solution) explodes in some regions and remains bounded in some others as the loss goes to 0 , might appear. In this paper, we report various mathematical results on the properties of NIMs and their applications. The topics are superlensing using complementary media (Section 2), cloaking using complementary media (Section 3), cloaking an object via anomalous localized resonance (Section 4), and the well-posedness and the finite speed propagation properties of electromagnetic waves in the time domain for media consisting of dispersive metamaterials (Section 5). Concerning the first three topics, refined results in comparison with the original works will be presented. Other aspects on NIMs, such as the stability of NIMs and cloaking a source via anamlous localized resonance, will be mentioned briefly in the last section (Section 6) in which we also discuss future directions. This report can be considered as a companion to the one in [27] written in 2015 in which superlensing using complementary media, cloaking using complementary media, and the stability of NIMs and cloaking a source via anomalous localized resonance are discussed in the spirit of the original works.

\section{Superlensing Using Complementary Media}

Superlensing using NIMs was suggested by Veselago in his seminal paper [45]. In this paper, he considered a slab lens with $\epsilon=\mu=-I$, where $I$ denotes the identity matrix, 
and investigated its lensing property using ray theory. Later, the study of cylindrical lenses in the two-dimensional quasistatic regime, the Veselago slab lens, cylindrical lenses, and spherical lenses in the finite frequency regime were respectively suggested and examined by Nicorovici, McPhedran, and Milton [37]; Pendry [39, 40]; and Pendry and Ramakrishna $[42,43]$ for constant isotropic objects.

In this section, we present superlensing schemes in the spirit of [23] in which we established superlensing using complementary media for arbitrary objects. The superlensing schemes in [23] are inspired by the ones suggested in [37, 39, 40, 43] but different from there. The lenses in [23] also have their roots from [22] in which complementary media were defined and investigated from mathematical point of views. It was shown later in [28] that the modification proposed in [23] in comparison with [37, 39, 40, 43] was necessary in order to achieve superlensing (see also Section 4).

We next mathematically describe superlensing using complementary media. Let $B_{r}$ denote the ball in $\mathbb{R}^{d}$ centered at the origin and of radius $r>0$. We first consider the quasistatic acoustic setting in a two-dimensional, bounded domain. To magnify $m$ times $(m>1)$ an arbitrary object in $B_{r_{0}}$ with $r_{0}>0$, one uses a lens consisting of two layers as follows. The first layer in $B_{r_{1}} \backslash B_{r_{0}}$ is characterized by the identity matrix $I$, and the second one in $B_{r_{2}} \backslash B_{r_{1}}$ is characterized by the matrix $-I$. Here $r_{1}$ and $r_{2}$ are defined by

$$
r_{1}=m^{1 / 2} r_{0} \quad \text { and } \quad r_{2}=m r_{0} .
$$

Different choices for $r_{1}$ and $r_{2}$ are possible. Nevertheless, there are some restrictions on them. In particular, $r_{1} / r_{0}$ cannot be too close to 1 (see Remark 2.2). The choice considered in (2.1) has the advantage that the system is somehow stable for small loss (see (2.3)) and our proof of superlensing is quite simple in this case.

Assume that the object inside $B_{r_{0}}$ is characterized by a symmetric, uniformly elliptic, matrix-valued function $a$, i.e., for some constant $\Lambda \geq 1$,

$$
\Lambda^{-1}|\xi|^{2} \leq a(x) \xi \cdot \xi \leq \Lambda|\xi|^{2} \text { for a.e. } x \in B_{r_{0}} \text { and for } \xi \in \mathbb{R}^{2}
$$

and the medium outside $B_{r_{2}}$ is homegeneous and, hence, is characterized by the identity matrix $I$. Then, with the loss being described by a small, positive parameter $\delta$, the medium considered is characterized by $A_{\delta}:=s_{\delta} A,{ }^{1}$ where

$$
A=\left\{\begin{array}{l}
a \text { in } B_{r_{0}}, \\
I \text { otherwise, }
\end{array} \quad \text { and } \quad s_{\delta}=\left\{\begin{array}{cl}
-1-i \delta & \text { in } B_{r_{2}} \backslash B_{r_{1}}, \\
1 & \text { otherwise, }
\end{array} \text { for } \delta \geq 0 .\right.\right.
$$

The superlensing property of the considered lens is confirmed by the following theorem.

Theorem 2.1 Let $0<\delta<1, \Omega$ be a smooth, bounded, open subset of $\mathbb{R}^{2}, f \in L^{2}(\Omega)$, and set $r_{3}=r_{2}^{2} / r_{1}$. Assume that $B_{r_{3}} \subset \subset \Omega$ and supp $f \subset \Omega \backslash B_{r_{3}}$, and let $u_{\delta} \in H_{0}^{1}(\Omega)$ be the unique solution of the equation

$$
\operatorname{div}\left(A_{\delta} \nabla u_{\delta}\right)=f \text { in } \Omega
$$

We have

$$
\left\|u_{\delta}-\hat{u}\right\|_{H^{1}(\Omega)} \leq C \delta^{1 / 2}\|f\|_{L^{2}(\Omega)}
$$

and

$$
\left\|u_{\delta}-\hat{u}\right\|_{H^{1}\left(\Omega \backslash B_{r_{3}}\right)} \leq C \delta\|f\|_{L^{2}(\Omega)},
$$

\footnotetext{
${ }^{1} A_{0}$ plays the role of $\mathbf{A}$ in (1.2).
} 
for some positive constant $C$ independent of $f$ and $\delta$. In particular,

$$
u_{\delta} \rightarrow \hat{u} \text { in } H^{1}\left(\Omega \backslash B_{r_{3}}\right) \text { as } \delta \rightarrow 0 .
$$

Here $\hat{u} \in H_{0}^{1}(\Omega)$ is the unique solution of the equation

$$
\operatorname{div}(\hat{A} \nabla \hat{u})=f \text { in } \Omega, \text { where } \hat{A}=\left\{\begin{array}{cl}
a(\cdot / m) & \text { in } B_{r_{2}}, \\
I & \text { otherwise. }
\end{array}\right.
$$

Proof We first prove (2.3). The key idea of its proof is to construct a solution $u_{0} \in H_{0}^{1}(\Omega)$ to the equation $\operatorname{div}\left(A_{0} \nabla u_{0}\right)=f$ in $\Omega$. To motivate the construction of $u_{0} \in H_{0}^{1}(\Omega)$ below, we first assume that there exists such a solution $u_{0}$. Let $u_{1,0}$ be the reflection of $u_{0}$ in $B_{r_{2}}$ through $\partial B_{r_{2}}$ via the Kelvin transform $F$ associated with $\partial B_{r_{2}}$, i.e.,

$$
u_{1,0}(x)=u_{0} \circ F^{-1} \text { for } x \in \mathbb{R}^{2} \backslash B_{r_{2}} \text {, where } F(x):=r_{2}^{2} x /|x|^{2} \text { for } x \in \mathbb{R}^{2} .
$$

Note that $F$ respectively transforms $\partial B_{r_{1}}$ onto $\partial B_{r_{3}}$ and $\partial B_{r_{0}}$ onto $\partial B_{r_{2}}$; the constant $r_{3}$ appears naturally here. Since $\Delta u_{0}=0$ in $B_{r_{2}} \backslash \bar{B}_{r_{1}}$ and in $B_{r_{1}}$, it follows that

$$
\Delta u_{1,0}=0 \text { in } B_{r_{3}} \backslash \bar{B}_{r_{2}} \text { and in } \mathbb{R}^{2} \backslash \bar{B}_{r_{3}} .
$$

Moreover, by taking into account the continuity $u_{0}$ and its flux on $\partial B_{r_{2}}$ and $\partial B_{r_{3}}$, we have

$$
u_{1,0}=u_{0} \quad \text { and } \quad \partial_{r} u_{1,0}=-\left.\partial_{r} u_{0}\right|_{i n t}=\left.\partial_{r} u_{0}\right|_{\text {ext }} \text { on } \partial B_{r_{2}}
$$

and

$$
\left.u_{1,0}\right|_{\text {ext }}=\left.u_{1,0}\right|_{\text {int }} \quad \text { and }\left.\quad \partial_{r} u_{1,0}\right|_{\text {ext }}=-\left.\partial_{r} u_{1,0}\right|_{\text {int }} \text { on } \partial B_{r_{3}} .
$$

Here and in what follows, for a smooth, bounded, open subset $D$ of $\mathbb{R}^{d}$, on its boundary $\partial D$, $\left.u\right|_{\text {ext }}$ and $\left.u\right|_{\text {int }}$ denote the restriction of $u$ in $\mathbb{R}^{d} \backslash \bar{D}$ and the restriction of $u$ in $D$, respectively, for an appropriate function $u$. We also denote $[u]$ as the quantity $\left.u\right|_{\text {ext }}-\left.u\right|_{\text {int }}$ on $\partial D$ and use similar notations for $A \nabla u \cdot v$ for an appropriate function $u$ where $A$ is a matrix and $v$ is the unit normal vector on $\partial D$ directed to the exterior of $D$.

Let $u_{2,0}$ be the reflection of $u_{1,0}$ in $B_{r_{3}}$ through $\partial B_{r_{3}}$ via the Kelvin transform $G$ associated with $\partial B_{r_{3}}$, i.e.,

$$
u_{2,0}(x)=u_{1,0} \circ G^{-1} \text { for } x \in B_{r_{3}}, \text { where } G(x):=r_{3}^{2} x /|x|^{2} \text { for } x \in \mathbb{R}^{2} .
$$

We then have

$$
\begin{gathered}
\operatorname{div}\left(\hat{A} \nabla u_{2,0}\right)=0 \text { in } B_{r_{3}} \\
u_{2,0}=\left.u_{1,0}\right|_{\text {int }} \quad \text { and } \quad \partial_{r} u_{2,0}=\left.\partial_{r} u_{1,0}\right|_{\text {int }} \text { on } \partial B_{r_{3}} .
\end{gathered}
$$

The definition of $\hat{A}$ in $B_{r_{3}}$ appears naturally here. Since $\hat{A}=I$ in $B_{r_{3}} \backslash B_{r_{2}}$ by the choices of $r_{1}$ and $r_{2}\left(G \circ F\left(\partial B_{r_{0}}\right)=\partial B_{m r_{0}}=\partial B_{r_{2}}\right)$, it follows from (2.8) that

$$
\Delta u_{2,0}=0 \text { in } B_{r_{3}} .
$$

Set

Then,

$$
w_{0}=\left\{\begin{array}{cl}
u_{0} & \text { in } \Omega \backslash \bar{B}_{r_{2}}, \\
u_{2,0} & \text { in } B_{r_{2}} .
\end{array}\right.
$$

$$
\operatorname{div}\left(\hat{A} \nabla w_{0}\right)=f \text { in } \Omega \backslash \partial B_{r_{2}}, \quad\left[w_{0}\right]=0 \text { on } \partial B_{r_{2}}, \quad \text { and } \quad\left[\hat{A} \nabla w_{0} \cdot v\right]=0 \text { on } \partial B_{r_{2}} .
$$

It follows that $w_{0} \in H_{0}^{1}(\Omega)$ is a solution of

$$
\operatorname{div}\left(\hat{A} \nabla w_{0}\right)=f \text { in } \Omega .
$$


We derive that

$$
\hat{u}=w_{0} \text { in } \Omega \text {. }
$$

Inspired by the heuristic arguments above, we define

$$
u_{0}=\left\{\begin{array}{cl}
\hat{u} & \text { in } \Omega \backslash B_{r_{2}}, \\
\hat{u} \circ F & \text { in } B_{r_{2}} \backslash B_{r_{1}}, \\
\hat{u} \circ G \circ F=\hat{u}(m \cdot) & \text { in } B_{r_{0}} .
\end{array}\right.
$$

It is clear from the definition of $\hat{u}$ that

$$
\Delta u_{0}=f \text { in }\left(\Omega \backslash \bar{B}_{r_{2}}\right) \cup\left(B_{r_{2}} \backslash \bar{B}_{r_{1}}\right) \quad \text { and } \quad \operatorname{div}\left(a \nabla u_{0}\right)=0 \text { in } B_{r_{1}} .
$$

Moreover, one can check that

$$
\left[u_{0}\right]=0 \quad \text { and } \quad\left[s_{0} A \nabla u_{0} \cdot v\right]=0 \text { on }\left(\partial B_{r_{2}} \cup \partial B_{r_{1}}\right) .
$$

Combining (2.12), (2.13), and (2.14) yields that $u_{0} \in H_{0}^{1}(\Omega)$ is a solution of the equation $\operatorname{div}\left(A_{0} \nabla u_{0}\right)=f$ in $\mathbb{R}^{2}$.

We have

$$
\operatorname{div}\left(A_{\delta} \nabla\left(u_{\delta}-u_{0}\right)\right)=-\operatorname{div}\left(\left(A_{\delta}-A_{0}\right) \nabla u_{0}\right)=i \delta \operatorname{div}\left(\chi_{B_{r_{2}} \backslash B_{r_{1}}} \nabla u_{0}\right) \text { in } \Omega,
$$

where $\chi_{D}$ denotes the characteristic function of a given subset $D$ of $\mathbb{R}^{2}$. Applying (2.21) of Lemma 2.1 below, we have

$$
\left\|u_{\delta}-u_{0}\right\|_{H^{1}(\Omega)} \leq C\left\|\nabla u_{0}\right\|_{L^{2}\left(B_{r_{2}} \backslash B_{r_{1}}\right)} \leq C\|f\|_{L^{2}(\Omega)} .
$$

This yields, by (2.15) and (2.21) of Lemma 2.1 again,

$$
\left\|u_{\delta}-u_{0}\right\|_{H^{1}(\Omega)} \leq C \delta^{1 / 2}\|f\|_{L^{2}(\Omega)},
$$

which is (2.3).

We next establish (2.4). Similar to the definition of $u_{1,0}$ and $u_{2,0}$, we define $u_{1, \delta}$ in $\mathbb{R}^{2} \backslash \bar{B}_{r_{2}}$ and $u_{2, \delta}$ in $B_{r_{3}}$ as follows

$$
u_{1, \delta}=u_{\delta} \circ F^{-1} \text { in } \mathbb{R}^{2} \backslash \bar{B}_{r_{2}} \quad \text { and } \quad u_{2, \delta}=u_{1, \delta} \circ G^{-1} \text { in } B_{r_{3}} .
$$

As above, one can verify that

$$
\Delta u_{1, \delta}=0 \text { in } B_{r_{3}} \backslash \bar{B}_{r_{2}}, \quad u_{1, \delta}=u_{\delta} \text { on } \partial B_{r_{2}}, \quad(1+i \delta) \partial_{r} u_{1, \delta}=\left.\partial_{r} u_{\delta}\right|_{\text {ext }} \text { on } \partial B_{r_{2}} \text {, }
$$

$\operatorname{div}\left(\hat{A} \nabla u_{2, \delta}\right)=0$ in $B_{r_{3}}, \quad u_{2, \delta}=u_{1, \delta}$ on $\partial B_{r_{3}}, \quad$ and $\quad \partial_{r} u_{2, \delta}=\left.(1+i \delta) \partial_{r} u_{1, \delta}\right|_{\text {int }}$ on $\partial B_{r_{3}}$.

Define

One can check that

$$
\hat{u}_{\delta}=\left\{\begin{array}{cl}
u_{\delta} & \text { in } \Omega \backslash B_{r_{3}}, \\
u_{\delta}-\left(u_{1, \delta}-u_{2, \delta}\right) & \text { in } B_{r_{3}} \backslash B_{r_{2}}, \\
u_{2, \delta} & \text { in } B_{r_{2}} .
\end{array}\right.
$$

$$
\operatorname{div}\left(\hat{A} \nabla\left(\hat{u}_{\delta}-\hat{u}_{0}\right)\right)=0 \text { in } \Omega \backslash\left(\partial B_{r_{2}} \cup \partial B_{r_{3}}\right) .
$$

Moreover, by (2.16) and (2.17), one has

$\left[\hat{u}_{\delta}-\hat{u}_{0}\right]=u_{\delta}-u_{1, \delta}=0, \quad\left[\hat{A} \nabla\left(u_{\delta}-\hat{u}_{0}\right) \cdot e_{r}\right]=\hat{A} \nabla\left(\left.u_{\delta}\right|_{\text {ext }}-u_{1, \delta}\right) \cdot e_{r}=\left.i \delta \partial_{r} u_{1, \delta}\right|_{\text {int }}$ on $\partial B_{r_{2}}$, and

$\left[\hat{u}_{\delta}-\hat{u}_{0}\right]=u_{1, \delta}-u_{2, \delta}=0, \quad\left[\hat{A} \nabla\left(u_{\delta}-\hat{u}_{0}\right) \cdot e_{r}\right]=\partial_{r}\left(\left.u_{1, \delta}\right|_{\text {int }}-u_{2, \delta}\right)=-\left.i \delta \partial_{r} u_{1, \delta}\right|_{\text {int }}$ on $\partial B_{r_{3}}$. 
From Lemma 2.2 below, it follows that

$$
\begin{aligned}
\left\|\hat{u}_{\delta}-\hat{u}_{0}\right\|_{H^{1}\left(\Omega \backslash\left(\partial B_{r_{2}} \cup \partial B_{r_{3}}\right)\right)} & \leq C\left(\left\|\left.\delta \partial_{r} u_{1, \delta}\right|_{\text {int }}\right\|_{H^{-1 / 2}\left(\partial B_{r_{3}}\right)}+\left\|\left.\delta \partial_{r} u_{1, \delta}\right|_{\text {ext }}\right\|_{H^{-1 / 2}\left(\partial B_{r_{2}}\right)}\right) \\
& \leq C \delta\|f\|_{L^{2}(\Omega)} .
\end{aligned}
$$

In the last inequality, we use (2.3). Since $\hat{u}_{\delta}=u_{\delta}$ in $\Omega \backslash \bar{B}_{r_{3}}$, assertion (2.4) follows.

The proof is complete.

Remark 2.1 Assertion (2.5) in a more general setting, the setting of complementary media, is given in [22]. In [22], $s_{\delta}$ is defined by $-1+i \delta$ in $B_{r_{2}} \backslash B_{r_{1}}$; nevertheless, this point is not essential. The proof of (2.3) also has its roots from [22]. The idea is to use reflections to derive Cauchy's problems from the original equation with sign-changing coefficients and then use the unique continuation principle; see, e.g., [41]. This can be applied to a general structure via the change of variable rule; see Lemma 2.3 below. Assertion (2.4) is new in comparison with [22] whose method only yields $\delta^{1 / 2}$ instead of $\delta$ as the rate of the convergence. The key ingredient in the proof is the introduction of the auxiliary function $\hat{u}_{\delta}$. This auxiliary function was introduced in the technique of removing localized singularity by the author to handle the localized resonance associated with NIMs in cloaking and superlensing applications; see our previous work [23, 25], Section 3, and Remark 2.2. Interestingly, it is also useful even in stable cases for improving the convergence rate. The motivation of (2.4) comes from simulations obtained in the master project of Droxler at EPFL under the supervision of Hesthaven and the author.

Remark 2.2 The choice of $r_{1}$ and $r_{2}$ in (2.1) is not strict for ensuring (2.5). In previous work [23], we showed that it is possible to choose

$$
r_{1}=m^{1 / 4} r_{0} \quad \text { and } \quad r_{2}=m^{1 / 2} r_{1}
$$

In fact, the approach in [23] also works for the choice

$$
r_{1} \geq m^{1 / 4} r_{0} \quad \text { and } \quad r_{2}=m^{1 / 2} r_{1}
$$

Instead of introducing $\hat{u}$ as in (2.18), we define $\hat{u}_{\delta}$ as follows

$$
\hat{u}_{\delta}=\left\{\begin{array}{cl}
u_{\delta} & \text { in } \Omega \backslash B_{r_{3}}, \\
u_{\delta}-\left(u_{1, \delta}-u_{2, \delta}\right) & \text { in } B_{r_{3}} \backslash B_{m r_{0}}, \\
u_{2, \delta} & \text { in } B_{m r_{0}} .
\end{array}\right.
$$

Recall that, if $v \in H^{1}\left(B_{R_{3}} \backslash B_{R_{1}}\right)$ satisfies $\Delta v=0$ in $B_{R_{3}} \backslash B_{R_{1}}$ for $0<R_{1}<R_{2}<R_{3}$, then

$$
\begin{aligned}
& \|v\|_{H^{1 / 2}\left(\partial B_{R_{2}}\right)}+\left\|\partial_{r} v\right\|_{H^{-1 / 2}\left(\partial B_{R_{2}}\right)} \\
\leq & C\left(\|v\|_{H^{1 / 2}\left(\partial B_{R_{1}}\right)}+\left\|\partial_{r} v\right\|_{H^{-1 / 2}\left(\partial B_{R_{1}}\right)}\right)^{\alpha}\left(\|v\|_{H^{1 / 2}\left(\partial B_{R_{3}}\right)}+\left\|\partial_{r} v\right\|_{H^{-1 / 2}\left(\partial B_{R_{3}}\right)}\right)^{1-\alpha}
\end{aligned}
$$


with $\alpha=\ln \left(R_{3} / R_{2}\right) / \ln \left(R_{3} / R_{1}\right)^{2}$. Using this inequality, one can prove that

$$
\left\|u_{1, \delta}-u_{\delta}\right\|_{H^{1 / 2}\left(\partial B_{m r_{0}}\right)}+\left\|\partial_{r}\left(u_{1, \delta}-u_{\delta}\right)\right\|_{H^{-1 / 2}\left(\partial B_{m r_{0}}\right)} \leq C \delta^{\alpha}\|v\|_{H^{1}\left(\Omega \backslash B_{r_{3}}\right)},
$$

with $\alpha=\ln \left(r_{3} / r_{2}\right) / \ln \left(r_{3} / r_{1}\right)$ which is greater than or equal to $1 / 2$ by (2.19) and the fact that $r_{3}=r_{2}^{2} / r_{1}$. Applying the approach used in the proof of (2.3), one can reach (2.5) in the case in which $\alpha>1 / 2$, which is equivalent to $r_{1}>m^{1 / 4} r_{0}$. The case in which $\alpha=1 / 2$, corresponding to the choice $r_{1}=m^{1 / 4} r_{0}$, requires further arguments; in this case, the convergence in (2.5) is replaced by the weak convergence. The interested reader can find the details in [23, the proof of (2.36)].

In the proof of Theorem 2.1, we used the following stability result on $u_{\delta}$.

Lemma 2.1 Let $d \geq 2, \delta_{0}>0,0<r_{1}<r_{2}$, $\Omega$ be a smooth, open subset of $\mathbb{R}^{d}$ with $B_{r_{2}} \subset \subset \Omega$, let $A$ be a uniformly elliptic, matrix-valued function defined in $\Omega$, and let $g \in H^{-1}(\Omega)^{3}$. Set $A_{\delta}=s_{\delta} A$, where $s_{\delta}$ is defined in (2.2). For $0<\delta<\delta_{0}$, there exists a unique solution $v_{\delta} \in H_{0}^{1}(\Omega)$ of

$$
\operatorname{div}\left(A_{\delta} \nabla v_{\delta}\right)=g \text { in } \Omega
$$

Moreover,

$$
\left\|v_{\delta}\right\|_{H^{1}(\Omega)}^{2} \leq \frac{C}{\delta}\left|\int_{\Omega} g \bar{v}_{\delta}\right|
$$

and

$$
\left\|v_{\delta}\right\|_{H^{1}(\Omega)}^{2} \leq \frac{C}{\delta}\left|\mathfrak{s} \int_{\Omega} g \bar{v}_{\delta}\right|+C\|g\|_{L^{2}(\Omega)}^{2} .
$$

Here $C$ denotes a positive constant independent of $g$ and $\delta$.

Here and in what follows, for a complex number $z$, we denote $\Im z$ and $\Re z$ as the imaginary part and the real part of $z$, respectively.

Remark 2.3 Various variants of Lemma 2.1 are used in the study of NIMs; see, e.g., [22, 28]. In inequality (2.22), one only considers the imaginary part of $\int_{\Omega} g \bar{v}_{\delta}$. This is useful for improvements on the convergent rate of cloaking effects considered later in Sections 3 and 4. Nevertheless, the proof presented below is quite standard and in the same spirit.

Proof Multiplying the equation of $v_{\delta}$ by $\bar{v}_{\delta}$ (the conjugate of $v_{\delta}$ ), integrating by parts, and considering the imaginary part and the real part of the obtained expression, one has

$$
\left\|\nabla v_{\delta}\right\|_{L^{2}(\Omega)}^{2} \leq \frac{C}{\delta}\left|\int_{\Omega} g \bar{v}_{\delta}\right|
$$

This implies (2.21) by the Poincaré inequality.

${ }^{2}$ This inequality can be obtained from the following representation of $v$ in $B_{R_{3}} \backslash B_{R_{1}}$ :

$$
v(r, \vartheta)=a_{0}+b_{0} \ln r+\sum_{n=1}^{\infty} \sum_{ \pm}\left(a_{n, \pm} r^{n}+b_{n, \pm} r^{-n}\right) e^{ \pm i n \vartheta} \text { in } B_{R_{3}} \backslash B_{R_{1}} .
$$

See also [31, Lemma 6].

${ }^{3} H^{-1}(\Omega)$ denotes the dual space of $H_{0}^{1}(\Omega)$. 
To obtain (2.22), we proceed as follows. Multiplying the equation of $v_{\delta}$ by $\bar{v}_{\delta}$, considering the imaginary part, one has

$$
\left\|\nabla v_{\delta}\right\|_{L^{2}\left(B_{r_{2}} \backslash B_{r_{1}}\right)}^{2} \leq \frac{C}{\delta}\left|\Im \int_{\Omega} g \bar{v}_{\delta}\right| .
$$

We claim that

$$
\left\|v_{\delta}\right\|_{L^{2}\left(B_{r_{2}} \backslash B_{r_{1}}\right)} \leq C\left(\left\|\nabla v_{\delta}\right\|_{L^{2}\left(B_{r_{2}} \backslash B_{r_{1}}\right)}+\|g\|_{L^{2}(\Omega)}\right) .
$$

Assuming this, we obtain

$$
\left\|v_{\delta}\right\|_{H^{1}\left(B_{r_{2}} \backslash B_{r_{1}}\right)} \leq C\left(\left\|\nabla v_{\delta}\right\|_{L^{2}\left(B_{r_{2}} \backslash B_{r_{1}}\right)}+\|g\|_{L^{2}(\Omega)}\right) .
$$

This implies, by the trace theory,

$$
\left\|v_{\delta}\right\|_{H^{1 / 2}\left(\partial B_{r_{2}} \cup \partial B_{r_{1}}\right)} \leq C\left(\left\|\nabla v_{\delta}\right\|_{L^{2}\left(B_{r_{2}} \backslash B_{r_{1}}\right)}+\|g\|_{L^{2}(\Omega)}\right) .
$$

Using the equation of $v_{\delta}$ in $\Omega \backslash B_{r_{3}}$ and in $B_{r_{1}}$, we derive from the standard theory of elliptic equations that

$$
\left\|v_{\delta}\right\|_{\left.H^{1}\left(\left(\Omega \backslash B_{r_{2}}\right)\right) \cup B_{r_{1}}\right)} \leq C\left(\left\|\nabla v_{\delta}\right\|_{L^{2}\left(B_{r_{2}} \backslash B_{r_{1}}\right)}+\|g\|_{L^{2}(\Omega)}\right),
$$

and the conclusion follows from (2.23).

It remains to prove (2.24), which we establish by contradiction. Suppose that there exist a sequence $\delta_{n} \rightarrow 0$ (by (2.21)) and a sequence $g_{n} \rightarrow 0$ in $L^{2}(\Omega)$ such that

$$
1=\left\|v_{\delta_{n}}\right\|_{L^{2}\left(B_{r_{2}} \backslash B_{r_{1}}\right)} \geq n\left(\left\|\nabla v_{\delta_{n}}\right\|_{L^{2}\left(B_{r_{2}} \backslash B_{r_{1}}\right)}+\left\|g_{n}\right\|_{L^{2}(\Omega)}\right),
$$

where $v_{\delta_{n}}$ is the solution corresponding to $\delta_{n}$ and $g_{n}$. By the trace theory, one has

$$
\left\|v_{\delta_{n}}\right\|_{H^{1 / 2}\left(\partial B_{r_{2}} \cup \partial B_{r_{1}}\right)} \leq C
$$

for some positive constant $C$ independent of $n$. This in turn implies that

$$
\left\|v_{\delta_{n}}\right\|_{H^{1}(\Omega)} \leq C \text {. }
$$

Without loss of generality, one can assume that $v_{\delta_{n}}$ converges to $v_{0} \in H_{0}^{1}(\Omega)$ weakly in $H^{1}(\Omega)$ and strongly in $L^{2}\left(B_{r_{2}} \backslash B_{r_{1}}\right)$. Moreover,

$$
\operatorname{div}\left(A_{0} \nabla v_{0}\right)=0 \text { in } \Omega \text { and } \quad v_{0} \text { is constant in } B_{r_{2}} \backslash B_{r_{1}} .
$$

Since, by multiplying the equation of $v_{0}$ with $\bar{v}_{0}$ and integrating by parts,

$$
\int_{\Omega} A_{0} \nabla v_{0} \cdot \nabla v_{0}=0
$$

and $v_{0}$ is constant in $B_{r_{2}} \backslash B_{r_{1}}$, it follows that

$$
\int_{\Omega}\left|\nabla v_{0}\right|^{2}=0
$$

We derive that $v_{0}=0$ in $\Omega$ since $v_{0} \in H_{0}^{1}(\Omega)$. This contradicts the fact that $\int_{B_{r_{2}} \backslash B_{r_{1}}}\left|v_{0}\right|^{2}=$ $\lim _{n \rightarrow+\infty} \int_{B_{r_{2}} \backslash B_{r_{1}}}\left|v_{\delta_{n}}\right|^{2}=1$.

The following lemma is standard and was used in the proof of Theorem 2.1.

Lemma 2.2 Let $d=2,3, \Omega$ be a smooth, open subset of $\mathbb{R}^{d}$, and let A be a symmetric, uniformly elliptic, matrix-valued function defined in $\Omega$, and let $f \in L^{2}(\Omega)$. Let $D \subset \subset \Omega$ 
be a smooth, bounded, open subset of $\mathbb{R}^{d}$, let $g \in H^{1 / 2}(\partial D)$, and $h \in H^{-1 / 2}(\partial D)$. Assume that $v \in H^{1}(\Omega \backslash \partial D)$ satisfies

$$
\left\{\begin{array}{cl}
\operatorname{div}(A \nabla v)=f & \text { in } \Omega \backslash \partial D \\
{[v]=g \text { and }[A \nabla v \cdot v]=h} & \text { on } \partial D \\
v=0 & \text { on } \partial \Omega
\end{array}\right.
$$

Then,

$$
\|v\|_{H^{1}(\Omega \backslash \partial D)} \leq C\left(\|f\|_{L^{2}(\Omega)}+\|g\|_{H^{1 / 2}(\partial D)}+\|h\|_{H^{-1 / 2}(\partial D)}\right),
$$

for some positive constant $C$ depending only on $D, \Omega$, and the ellipticity of $A$.

The approach used in the proof of Theorem 2.1 can be extended to the finite frequency regime as well as higher dimensions. The additional tool is the following change of variables rule; see, e.g., [22, Lemma 2].

Lemma 2.3 Let $d \geq 2, D_{1} \subset \subset D_{2} \subset \subset D_{3}$ be three smooth, bounded, open subsets of $\mathbb{R}^{d}$. Let $a \in\left[L^{\infty}\left(D_{2} \backslash \bar{D}_{1}\right)\right]^{d \times d}, \sigma \in L^{\infty}\left(D_{2} \backslash D_{1}\right)$, and let $\mathcal{T}$ be a bijective from $D_{2} \backslash \bar{D}_{1}$ onto $D_{3} \backslash \bar{D}_{2}$ such that $\mathcal{T} \in C^{1}\left(\bar{D}_{2} \backslash D_{1}\right)$ and $\mathcal{T}^{-1} \in C^{1}\left(\bar{D}_{3} \backslash D_{2}\right)$. Assume that $u \in H^{1}\left(D_{2} \backslash D_{1}\right)$ and set $v=u \circ \mathcal{T}^{-1}$. Then

$$
\operatorname{div}(a \nabla u)+\sigma u=f \text { in } D_{2} \backslash D_{1},
$$

for some $f \in L^{2}\left(D_{2} \backslash D_{1}\right)$, if and only if

$$
\operatorname{div}\left(\mathcal{T}_{*} a \nabla v\right)+\mathcal{T}_{*} \sigma v=\mathcal{T}_{*} f \text { in } D_{3} \backslash D_{2} .
$$

Assume in addition that $\mathcal{T}(x)=x$ on $\partial D_{2}$. Then

$$
v=u \quad \text { and } \quad \mathcal{T}_{*} a \nabla v \cdot v=-a \nabla u \cdot v \text { on } \partial D_{2} .
$$

Here

$$
\mathcal{T}_{*} a(y)=\frac{D \mathcal{T}(x) a(x) \nabla \mathcal{T}(x)^{T}}{|\operatorname{det} \nabla \mathcal{T}(x)|} \text { and } \mathcal{T}_{*} \sigma(y)=\frac{\sigma(x)}{|\operatorname{det} \nabla \mathcal{T}(x)|}, \quad \text { where } x=\mathcal{T}^{-1}(y) .
$$

Let $a$ be a symmetric, uniformly elliptic, matrix-valued function and $\sigma$ be a bounded complex function both defined in $B_{r_{0}}$ such that $\Re \sigma>c>0$ and $\Im \Sigma \geq 0$ in $B_{r_{0}}$ for some $c>0$. Assuming (2.1), we have the following result which is a variant of Theorem 2.1 in the finite frequency regime in both two and three dimensions.

Theorem 2.2 Let $d=2,3,0<\delta<1, k>0, R_{0}>r_{3}, f \in L^{2}\left(\mathbb{R}^{d}\right)$, and set $r_{3}=r_{2}^{2} / r_{1}$. Assume that supp $f \subset B_{R_{0}} \backslash B_{r_{3}}$, and let $u_{\delta}$ be the unique outgoing solution of the equation

$$
\operatorname{div}\left(A_{\delta} \nabla u_{\delta}\right)+k^{2} \Sigma_{\delta}=f \text { in } \mathbb{R}^{d},
$$

where $\left(A_{\delta}, \Sigma_{\delta}\right)=\left(s_{\delta} A, \bar{s}_{\delta} \Sigma\right)$ and

$$
A, \Sigma=\left\{\begin{array}{cl}
a, \sigma & \text { in } B_{r_{0}}, \\
F_{*}^{-1} I, F_{*}^{-1} 1 & \text { in } B_{r_{2}} \backslash B_{r_{1}}, \\
I, 1 & \text { otherwise, }
\end{array} \quad \text { and } \quad s_{\delta}=\left\{\begin{array}{cl}
-1-i \delta \text { in } B_{r_{2}} \backslash B_{r_{1}}, \\
1 & \text { otherwise. }
\end{array}\right.\right.
$$

We have

$$
\left\|u_{\delta}-\hat{u}\right\|_{H^{1}\left(B_{R}\right)} \leq C_{R} \delta^{1 / 2}\|f\|_{L^{2}\left(\mathbb{R}^{d}\right)}
$$


and

$$
\left\|u_{\delta}-\hat{u}\right\|_{H^{1}\left(B_{R} \backslash B_{r_{3}}\right)} \leq C_{R} \delta\|f\|_{L^{2}\left(\mathbb{R}^{d}\right)},
$$

for some positive constant $C_{R}$ independent of $f$ and $\delta$. In particular,

$$
u_{\delta} \rightarrow \hat{u} \text { in } H_{\mathrm{loc}}^{1}\left(\mathbb{R}^{d} \backslash B_{r_{3}}\right) \text { as } \delta \rightarrow 0 .
$$

Here, $\hat{u}$ is the unique outgoing solution of the equation

$$
\operatorname{div}(\hat{A} \nabla \hat{u})+k^{2} \hat{\Sigma} \hat{u}=f \text { in } \mathbb{R}^{d}, \text { where } \hat{A}, \hat{\Sigma}=\left\{\begin{array}{cl}
m^{2-d} a(x / m), m^{-d} \sigma(x / m) & \text { in } B_{r_{2}}, \\
I, 1 & \text { otherwise. }
\end{array}\right.
$$

Recall that a solution $v \in H_{\mathrm{loc}}^{1}\left(\mathbb{R}^{d} \backslash B_{R}\right)$ of the equation

$$
\Delta v+k^{2} v=0 \text { in } \mathbb{R}^{d} \backslash B_{R},
$$

for some $R>0$, is said to satisfy the outgoing condition if

$$
\partial_{r} v-i k v=o\left(r^{-\frac{d-1}{2}}\right) \text { as } r=|x| \rightarrow+\infty .
$$

Proof The proof of Theorem 2.2 is similar to the one of Theorem 2.1 by using Lemma 2.3 and applying variants of Lemmas 2.1 and 2.2; see, e.g., [22, Lemma 1] or [30, Lemma 2.1] for variants of Lemma 2.1 which is of the form $\left|u_{\delta}\right|_{H^{1}\left(B_{R}\right)}^{2} \leq \frac{C_{R}}{\delta}\left|\Im \int_{\mathbb{R}^{d}} f \bar{u}_{\delta}-I\left(u_{\delta}\right)\right|+$ $C_{R}|f|_{L^{2}}^{2}$, where $I\left(u_{\delta}\right)=\lim _{R \rightarrow+\infty} \int_{\partial B_{R}} k\left|u_{\delta}\right|^{2}$. The details are left to the reader.

Remark 2.4 Superlensing using complementary media is justified mathematically for the electromagnetic wave [29]. The idea of using reflections is also useful in establishing superlensing using hyperbolic metamaterials, an interesting type of metamaterials, [7]

Remark 2.5 Using the change of variables in Lemma 2.3, one can design a general superlensing scheme in which one does not require $F$ (and also G) to be a Kelvin transform, and the lens is not required to be radially symmetric; see [22, Theorems 1 and 2 and Corollary 2] and [29, Theorem 2] for a discussion on the acoustic and electromagnetic settings, respectively.

\section{Cloaking Using Complementary Media}

Cloaking using complementary media was suggested by Lai et al. [19]. The idea is to cancel the effect of an object by its complementary medium, a concept considered in [42]; see [22] for a discussion of this concept from mathematical point of views. The study of cloaking using complementary media faces two difficulties. Firstly, this problem is unstable since the equations describing the phenomenon have sign-changing coefficients; hence, the ellipticity and the compactness are lost in general. Secondly, localized resonance might appear, as shown in simulations in [19].

Cloaking using complementary media was mathematically justified for acoustic waves [25] and for electromagnetic waves [31]. The schemes that were used in [25] and [31] are inspired by the work of Lai. et al. and the study of complementary concept in [22, 29]. Nevertheless, these schemes are different from the ones in [19]. The modification, mentioned below, is necessary, as shown in the acoustic setting in [28]; without the modification, cloaking might not be achieved (see also Section 4, Proposition 4.1, in particular, and the comments following). 
Let us describe how to cloak the region $B_{2 r_{2}} \backslash B_{r_{2}}$ for some $r_{2}>0$ in the spirit of [25]. We first consider the quasistatic regime. Assume that the cloaked region is characterized by a matrix $a$, which is symmetric and uniformly elliptic in $B_{2 r_{2}} \backslash B_{r_{2}}$. The cloaking device consists of two parts. The first one, in $B_{r_{2}} \backslash B_{r_{1}}$, makes use of reflecting complementary media to cancel the effect of the cloaked region, and the second one, in $B_{r_{1}}$, fills the space that "disappears" from the cancelation by the homogeneous medium. For the first part, we modify the strategy in [19]. Instead of $B_{2 r_{2}} \backslash B_{r_{2}}$, we consider $B_{r_{3}} \backslash B_{r_{2}}$ for some $r_{3}>0$ as the cloaked region in which the medium is given by the matrix

$$
a_{e}= \begin{cases}a & \text { in } B_{2 r_{2}} \backslash B_{r_{2}}, \\ I & \text { in } B_{r_{3}} \backslash B_{2 r_{2}} .\end{cases}
$$

We assume that

$$
a_{e} \in C^{1}\left(\bar{B}_{r_{3}} \backslash B_{r_{2}}\right) .
$$

The complementary medium in $B_{r_{2}} \backslash B_{r_{1}}$ is given by

$$
-\left(F^{-1}\right)_{*} a_{e}
$$

where $F: B_{r_{2}} \backslash \bar{B}_{r_{1}} \rightarrow B_{r_{3}} \backslash \bar{B}_{r_{2}}$ is the Kelvin transform with respect to $\partial B_{r_{2}}$. Concerning the second part, the medium in $B_{r_{1}}$ is given by

$$
\left(r_{3}^{2} / r_{2}^{2}\right)^{d-2} I
$$

which is also different from that suggested by Lai et al. [19]. The reason for this choice is to ensure that

$$
G_{*} F_{*} A=I \text { in } B_{r_{3}},
$$

where $A$ is defined in (3.4) below. In two dimensions, the medium in $B_{r_{1}}$ is $I$, as used by Lai et al. [19], while it is not $I$ in three dimensions. With the loss, the medium is characterized by $A_{\delta}:=s_{\delta} A$, where

$$
A=\left\{\begin{array}{cl}
a_{e} & \text { in } B_{r_{3}} \backslash B_{r_{2}}, \\
F_{*}^{-1} a_{e} & \text { in } B_{r_{2}} \backslash B_{r_{1}}, \\
\left(r_{3}^{2} / r_{2}^{2}\right)^{d-2} I & \text { in } B_{r_{1}}, \\
I & \text { otherwise, }
\end{array} \quad \text { and } s_{\delta}=\left\{\begin{array}{c}
-1-i \delta \text { in } B_{r_{2} \backslash B_{r_{1}},} \text { for } \delta \geq 0 . \\
1 \quad \text { otherwise, }
\end{array}\right.\right.
$$

Let $\Omega$ be a smooth bounded open subset of $\mathbb{R}^{d}$ with $B_{r_{3}} \subset \subset \Omega$, and let $f \in L^{2}(\Omega)$. Denote $u_{\delta}, \hat{u} \in H_{0}^{1}(\Omega)$, respectively, the unique solution of

$$
\operatorname{div}\left(A_{\delta} \nabla u_{\delta}\right)=f \text { in } \Omega \quad \text { and } \quad \Delta \hat{u}=f \text { in } \Omega .
$$

The cloaking property of this scheme is given in the following theorem.

Theorem 3.1 Let $d=2,3,0<\delta<1$, and $f \in L^{2}(\Omega)$ with supp $f \subset \Omega \backslash B_{r_{3}}$. Let $u_{\delta}, u \in H_{0}^{1}(\Omega)$ be the uniques solutions defined by (3.5). For any $0<\alpha<1$, there exists $\ell>0$, depending only on $r_{2}, \alpha$, and the ellipticity and the Lipschitz constants of $a_{e}$ such that if $r_{3}>\ell r_{2}$ then

$$
\left\|u_{\delta}\right\|_{H^{1}(\Omega)} \leq C \delta^{(\alpha-1) / 2}\|f\|_{L^{2}(\Omega)},
$$

and

$$
\left\|u_{\delta}-\hat{u}\right\|_{H^{1}\left(\Omega \backslash B_{r_{3}}\right)} \leq C \delta^{\alpha}\|f\|_{L^{2}(\Omega)},
$$


for some positive constant $C$ independent of $\delta$ and $f$. In particular, we have

$$
u_{\delta} \rightarrow \hat{u} \text { in } H^{1}\left(\Omega \backslash \bar{B}_{r_{3}}\right) \text { as } \delta \rightarrow 0 .
$$

For an observer outside $B_{r_{3}}$, the medium in $B_{r_{3}}$ given by $A_{\delta}$ looks as the homogeneous one by (3.6) for small $\delta$ : one has cloaking.

\section{Proof Set}

$$
\beta=(2+\alpha) / 3
$$

We have, by Lemma 2.1,

$$
\left\|u_{\delta}\right\|_{H^{1}(\Omega)} \leq C \operatorname{Data}(f, \delta),
$$

where

$$
\operatorname{Data}(f, \delta):=\frac{1}{\delta}\left|\mathfrak{I} \int_{\Omega} f \bar{u}_{\delta}\right|+\|f\|_{L^{2}(\Omega)} .
$$

As in the proof of Theorem 2.1, define $u_{1, \delta} \in H_{\mathrm{loc}}^{1}\left(\mathbb{R}^{d} \backslash B_{r_{2}}\right)$ and $u_{2, \delta} \in H^{1}\left(B_{r_{3}}\right)$ as follows

$$
u_{1, \delta}=u_{\delta} \circ F^{-1} \text { in } \mathbb{R}^{d} \backslash B_{r_{2}} \quad \text { and } \quad u_{2, \delta}=u_{1, \delta} \circ G^{-1}=u_{\delta} \circ F^{-1} \circ G^{-1} \text { in } B_{r_{3}} .
$$

We have, by Lemma 2.3,

$\operatorname{div}\left(A \nabla u_{1, \delta}\right)=0$ in $B_{r_{3}} \backslash B_{2 r_{2}}, \quad u_{1, \delta}=u_{\delta}$ on $\partial B_{r_{2}}$, and $(1+i \delta) A \nabla u_{1, \delta}=\left.A \nabla u_{\delta}\right|_{\text {ext }}$ on $\partial B_{r_{2}}$. Let $\mathcal{A}$ be a Lipschitz extension of $a_{e}$ in $B_{r_{3}}$ such that $\mathcal{A}(0)=I$ and let $w_{\delta} \in H_{0}^{1}\left(B_{r_{3}}\right)$ be such that

$$
\operatorname{div}\left(\mathcal{A} \nabla w_{\delta}\right)=0 \text { in } B_{r_{3}} \backslash \partial B_{r_{2}} \quad \text { and } \quad\left[\mathcal{A} \nabla w_{\delta} \cdot v\right]=i \delta A \nabla u_{1, \delta} \text { on } \partial B_{r_{2}} .
$$

Then

$$
\left\|w_{\delta}\right\|_{H^{1}\left(B_{r_{3}}\right)} \leq C \delta \operatorname{Data}(f, \delta)^{1 / 2} .
$$

Applying a three-sphere inequality [25, Lemma 1] to $\left(u_{1, \delta}-u_{\delta}\right) \chi_{B_{r_{3}} \backslash B_{r_{2}}}-w_{\delta}$ in $B_{r_{3}}{ }^{4}$ and using (3.11), we obtain, if $\ell$ is sufficiently large, that

$$
\left\|u_{\delta}-u_{1, \delta}\right\|_{H^{1 / 2}\left(\partial B_{2 r_{2}}\right)}+\left\|\left.\partial_{r}\left(u_{\delta}-u_{1, \delta}\right)\right|_{\text {ext }}\right\|_{H^{-1 / 2}\left(\partial B_{2 r_{2}}\right)} \leq C \delta^{\beta} \operatorname{Data}(f, \delta)^{1 / 2} .
$$

In the spirit of (2.20), we define

$$
\hat{u}_{\delta}=\left\{\begin{array}{cl}
u_{\delta} & \text { in } \Omega \backslash B_{r_{3}}, \\
u_{\delta}-\left(u_{1, \delta}-u_{2, \delta}\right) & \text { in } B_{r_{3}} \backslash B_{2 r_{2}}, \\
u_{2, \delta} & \text { in } B_{2 r_{2}} .
\end{array}\right.
$$

We have

$$
\begin{gathered}
\Delta\left(\hat{u}_{\delta}-\hat{u}\right)=0 \text { in } \Omega \backslash\left(\partial B_{r_{3}} \cup \partial B_{2 r_{2}}\right), \\
{\left[\hat{u}_{\delta}-\hat{u}\right]=0 \text { on } \partial B_{r_{3}}, \quad\left[\partial_{r}\left(\hat{u}_{\delta}-\hat{u}\right)\right]=-\left.i \delta \partial_{r} u_{1, \delta}\right|_{\text {int }} \text { on } \partial B_{r_{3}},}
\end{gathered}
$$

and

$$
\left[\hat{u}_{\delta}-\hat{u}\right]=u_{\delta}-u_{1, \delta}, \quad\left[\partial_{r}\left(u_{\delta}-\hat{u}\right)\right]=\partial_{r}\left(\left.u_{\delta}\right|_{\text {ext }}-u_{1, \delta}\right) \quad \text { on } \partial B_{2 r_{2}} .
$$

By Lemma 2.1, we obtain from (3.9) and (3.12) that

$$
\left\|\hat{u}_{\delta}-\hat{u}_{0}\right\|_{H^{1}(\Omega)} \leq C \delta^{\beta} \operatorname{Data}(f, \delta)^{1 / 2} .
$$

By (3.10), this implies, since $\beta>1 / 2$, that

$$
\left\|\hat{u}_{\delta}\right\|_{H^{1}\left(\Omega \backslash B_{r_{3}}\right)} \leq C\|f\|_{L^{2}(\Omega)} .
$$

\footnotetext{
${ }^{4}$ Recall that $\chi_{D}$ denotes the characteristic function of a subset $D$ of $\mathbb{R}^{d}$.
} 
We derive from (3.10) and (3.15) that

$$
\operatorname{Data}(f, \delta) \leq C \delta^{-1}\|f\|_{L^{2}(\Omega)}^{2}
$$

and from (3.14) and (3.15) that

$$
\left\|\hat{u}_{\delta}-\hat{u}\right\|_{H^{1}(\Omega)} \leq C \delta^{\beta-1 / 2}\|f\|_{L^{2}(\Omega)} .
$$

Up to this point, the analysis is in the spirit of [25], and we now add some new ingredients to derive the desired conclusions. We have, by (3.17),

$$
\left|\int_{\Omega} f \overline{\hat{u}}_{\delta}-\int_{\Omega} f \overline{\hat{u}}\right| \leq C \delta^{\beta-1 / 2}\|f\|_{L^{2}(\Omega)}^{2}
$$

and, by multiplying the equation of $\hat{u}$ with $\overline{\hat{u}}$ and considering the imaginary part,

$$
\Im \int_{\Omega} f \overline{\hat{u}}=0 .
$$

It follows from (3.10) that

$$
\operatorname{Data}(f, \delta) \leq C \delta^{\beta-3 / 2}\|f\|_{L^{2}(\Omega)}^{2} .
$$

From (3.14), we obtain

$$
\left\|\hat{u}_{\delta}-\hat{u}\right\|_{H^{1}(\Omega)} \leq C \delta^{3 \beta / 2-3 / 4}\|f\|_{L^{2}(\Omega)} .
$$

Repeating this process, one reaches, for $n \geq 1$, that

$$
\operatorname{Data}(f, \delta) \leq C_{n} \delta^{\beta\left(1+1 / 2+\cdots+1 / 2^{n-1}\right)-\left(1 / 2+\cdots+1 / 2^{n}\right)-1}\|f\|_{L^{2}(\Omega)}^{2}
$$

and

$$
\left\|\hat{u}_{\delta}-\hat{u}\right\|_{H^{1}(\Omega)} \leq C_{n} \delta^{\beta\left(1+1 / 2+\cdots+1 / 2^{n}\right)-\left(1 / 2+\cdots+1 / 2^{n+1}\right)}\|f\|_{L^{2}(\Omega)},
$$

where $C_{n}$ is a positive constant independent of $\delta$ and $f$. The conclusion follows by taking $n$ large enough.

Remark 3.1 One of the crucial steps of this proof is to introduce the function $\hat{u}$. In general $u_{1, \delta}-u_{\delta}$ explodes in the region $B_{r_{3}} \backslash B_{2 r_{2}}$. A numerical simulation of this fact is given in the work of Lai. et al. [19]. A mathematical illustration of this phenomenon can be seen from the explicit representation of $u_{\delta}-u_{1, \delta}$ in $B_{r_{3}} \backslash B_{2 r_{2}}$ using separation of variables; see [25, Proof of Theorem 1]. The definition of $\hat{u}$ is inspired by the concept of the normalizing energy used in the study of the Ginzburg-Landau equation; see, e.g., [4].

We next present the result in the finite frequency regime. Assume that the cloaked region is characterized by a matrix $a$ that is symmetric, uniformly elliptic and a bounded complex function $\sigma$ that satisfies $\Re \sigma>c>0$ and $\Im \sigma \geq 0$ both defined in $B_{2 r_{2}} \backslash B_{r_{2}}$. As in the spirit of the zero-frequency case, we consider the layer $B_{r_{3}} \backslash B_{r_{2}}$ as the cloaked region that is characterized by

$$
a_{e}, \sigma_{e}=\left\{\begin{array}{l}
a, \sigma \text { in } B_{2 r_{2} \backslash B_{r_{2}}}, \\
I, 1 \text { in } B_{r_{3}} \backslash B_{2 r_{2}} .
\end{array}\right.
$$

The cloaking device consists of two parts. The first one, the complementary layer in $B_{r_{2}} \backslash$ $B_{r_{1}}$, is characterized by

$$
-\left(F^{-1}\right)_{*} a_{e},-F_{*}^{-1} \sigma_{e} .
$$


Concerning the second part, the medium in $B_{r_{1}}$ is given by

$$
\left(r_{3}^{2} / r_{2}^{2}\right)^{d-2} I,\left(r_{3}^{2} / r_{2}^{2}\right)^{d}
$$

Again, the reason for this choice is to ensure

$$
G_{*} F_{*} A=I \text { and } G_{*} F_{*} \Sigma=1 \text { in } B_{r_{3}},
$$

where $A$ and $\Sigma$ are defined in (3.20). We will assume that (3.1) holds. Set $A_{\delta}:=s_{\delta} A$ and $\Sigma_{\delta}:=\bar{s}_{\delta} \Sigma$, where, for $\delta \geq 0$,

$$
A, \Sigma=\left\{\begin{array}{cl}
a_{e}, \sigma_{e} & \text { in } B_{r_{3}} \backslash B_{r_{2}}, \\
F_{*}^{-1} a_{e}, F_{*}^{-1} \sigma_{e} & \text { in } B_{r_{2}} \backslash B_{r_{1}}, \\
\left(r_{3}^{2} / r_{2}^{2}\right)^{d-2} I,\left(r_{3}^{2} / r_{2}^{2}\right)^{d} & \text { in } B_{r_{1}}, \\
I, 1 & \text { otherwise, }
\end{array} \quad \text { and } s_{\delta}=\left\{\begin{array}{cl}
-1-i \delta & \text { in } B_{r_{2}} \backslash B_{r_{1}}, \\
1 & \text { otherwise. }
\end{array}\right.\right.
$$

Let $k>0, f \in L^{2}\left(\mathbb{R}^{d}\right)$ with compact support and denote $u_{\delta}, \hat{u} \in H_{\mathrm{loc}}^{1}\left(\mathbb{R}^{d}\right)$, respectively, the unique outgoing solutions of

$$
\operatorname{div}\left(A_{\delta} \nabla u_{\delta}\right)+k^{2} \Sigma_{\delta} u_{\delta}=f \text { in } \mathbb{R}^{d} \quad \text { and } \quad \Delta \hat{u}+k^{2} \hat{u}=f \text { in } \mathbb{R}^{d} .
$$

Here is the variant of Theorem 3.1 for the finite frequency regime, which confirms the cloaking property of the scheme considered.

Theorem 3.2 Let $d=2,3, k>0,0<\delta<1, R_{0}>r_{3}, f \in L^{2}\left(\mathbb{R}^{d}\right)$ with supp $f \subset$ $B_{R_{0}} \backslash B_{r_{3}}$. Let $u_{\delta}, u \in H_{\mathrm{loc}}^{1}\left(\mathbb{R}^{d}\right)$ be the unique outgoing solutions defined by (3.21). For any $0<\alpha<1$, there exists $\ell>0$, depending only on $r_{2}, \alpha$, and the ellipticity and the Lipschitz. constants of $a_{e}$ such that if $r_{3}>\ell r_{2}$ then

$$
\left\|u_{\delta}\right\|_{H^{1}\left(B_{R}\right)} \leq C \delta^{(\alpha-1) / 2}\|f\|_{L^{2}\left(\mathbb{R}^{d}\right)},
$$

and

$$
\left\|u_{\delta}-\hat{u}\right\|_{H^{1}\left(B_{R} \backslash B_{r_{3}}\right)} \leq C \delta^{\alpha}\|f\|_{L^{2}\left(\mathbb{R}^{d}\right)},
$$

for some positive constant $C$ independent of $\delta$ and $f$. In particular, we have

$$
u_{\delta} \rightarrow \hat{u} \text { in } H_{\mathrm{loc}}^{1}\left(\mathbb{R}^{d} \backslash \bar{B}_{r_{3}}\right) \text { as } \delta \rightarrow 0 .
$$

Proof The proof of Theorem 3.2 is in the spirit of Theorem 3.1 with a crucial point being the establishment of (3.12) in the finite frequency regime. This can be done as follows. On one hand, we have, by [33, Theorem 2],

$$
\begin{aligned}
& \left\|u_{\delta}-u_{1, \delta}\right\|_{\mathbf{H}\left(\partial B_{2 r_{2}}\right)} \\
\leq & C\left\|\left.\partial_{r}\left(u_{\delta}-u_{1, \delta}\right)\right|_{\text {ext }}\right\|_{\mathbf{H}\left(\partial B_{r_{2}}\right)}^{\alpha}\left(\left\|u_{\delta}-u_{1, \delta}\right\|_{\mathbf{H}\left(\partial B_{4 r_{2}}\right)}+\left\|\left.u_{\delta}\right|_{\text {ext }}-u_{1, \delta}\right\|_{\mathbf{H}\left(\partial B_{r_{2}}\right)}\right)^{1-\alpha} .
\end{aligned}
$$

for some positive constant $\tau$ depending only on $r_{2}$ and the ellipticity and the Lipschitz of $a_{e}$. Here we denote

$$
\|v\|_{\mathbf{H}\left(\partial B_{r}\right)}:=\|v\|_{H^{1 / 2}\left(\partial B_{r}\right)}+\|A \nabla v \cdot v\|_{H^{-1 / 2}\left(\partial B_{r}\right)} .
$$

On the other hand, we obtain, by [31, Lemma 6], that

$$
\left\|u_{\delta}-u_{1, \delta}\right\|_{\mathbf{H}\left(\partial B_{4 r_{2}}\right)} \leq C_{k}\left\|u_{\delta}-u_{1, \delta}\right\|_{\mathbf{H}\left(\partial B_{2 r_{2}}\right)}^{\xi}\left\|u_{\delta}-u_{1, \delta}\right\|_{\mathbf{H}\left(\partial B_{r_{3}}\right)}^{1-\xi},
$$


where $\xi=\ln \left(r_{3} /\left(4 r_{2}\right)\right) / \ln \left(r_{3} /\left(2 r_{2}\right)\right)$. Combining (3.25) and (3.26) yields (3.12) if $\ell$ is sufficiently large. The rest of the proof is in the spirit of Theorem 2.1. The details are omitted.

Remark 3.2 Previous given proof of cloaking using complementary media [25] can be extended to the finite frequency regime. Nevertheless, the size of the cloaked object (the cloaked region) is small as $k$ is large. In [33], we extended the approach in [25] for the finite frequency regime in which the size of the object can be independent of the frequency $k$. In fact, we showed that there exists $\lambda_{0}>1$ depending on the ellipticity and the Lipschitz of $a_{e}$ such that one can cloak an object inside $B_{\lambda_{0} r_{2}} \backslash B_{r_{2}}$; nevertheless, $\lambda_{0}$ can be smaller than 2 but one can choose a large $r_{2}$ to compensate this. The proof given here is again in the spirit of the work [31] in which cloaking using complementary media for electromagnetic waves is investigated.

Remark 3.3 Using the change of variables in Lemma 2.3, one can design a general cloaking scheme in which one does not require $F$ (and also G) to be a Kelvin transform and the cloaking device is not necessary to be radially symmetric; see [31] for a discussion in the electromagnetic setting.

\section{Cloaking an Object via Anomalous Localized Resonance}

In this section, we present another cloaking technique using NIMs namely cloaking an object via anomalous localized resonance. The advantage of this cloaking technique over the one using complementary media is that the cloaking devices used here are independent of the cloaked object. This cloaking technique was suggested in [28] and inspired from [21, 24]. In the two-dimensional case, it was shown in [28] that the negative index layer of the lens considered in Section 2 can act like a cloaking device for a finite size object near by; see Fig. 1. More precisely, in the quasistatic regime, we have

Theorem 4.1 Let $d=2,0<r_{0}<r_{1}<r_{2}, x_{1} \in \partial B_{r_{1}}$, and $x_{2} \in \partial B_{r_{2}}$. Set $r_{3}:=r_{2}^{2} / r_{1}$ and $\mathcal{C}:=\left(B\left(x_{1}, r_{0}\right) \cap B_{r_{1}}\right) \cup\left(B\left(x_{2}, r_{0}\right) \cap\left(B_{r_{3}} \backslash B_{r_{2}}\right)\right)$, assume that $B_{r_{3}} \subset \subset \Omega$ and let $a_{c}$ be $a$ symmetric uniformly elliptic matrix-valued function defined in $\mathcal{C}$. Define

$$
A_{c}=\left\{\begin{array}{l}
a_{c} \text { in } \mathcal{C}, \\
I \text { otherwise, }
\end{array} \quad \text { and } s_{\delta}=\left\{\begin{array}{cc}
-1-i \delta & \text { in } B_{r_{2}} \backslash B_{r_{1}}, \\
1 & \text { otherwise. }
\end{array}\right.\right.
$$

Given $f \in L^{2}(\Omega)$ with supp $f \subset \Omega \backslash B_{r_{3}}$, let $u_{\delta}, \hat{u} \in H_{0}^{1}(\Omega)$, respectively, be the unique solution to the equations

$$
\operatorname{div}\left(A_{\delta} \nabla u_{\delta}\right)=f \text { in } \Omega \text { and } \Delta \hat{u}=f \text { in } \Omega .
$$

For any $0<\alpha<1$, there exists $r_{0}(\alpha)>0$ that depends only on $\alpha, r_{1}$, and $r_{2}$, such that if $r_{0}<r_{0}(\alpha)$ then

$$
\left\|u_{\delta}-\hat{u}\right\|_{H^{1}\left(\Omega \backslash B_{r_{3}}\right)} \leq C \delta^{\alpha}\|f\|_{L^{2}(\Omega)},
$$

where $C$ is a positive constant independent of $f, \delta, r_{0}, x_{1}$, and $x_{2}$.

Proof Set

$$
\beta=(2+\alpha) / 3 .
$$


Fig. 1 The red lens layer will cloak the blue region $\mathcal{C}$. An observer outside $B_{r_{3}}$ $\left(r_{3}=r_{2}^{2} / r_{1}\right)$ sees neither the red layer nor the blue regions

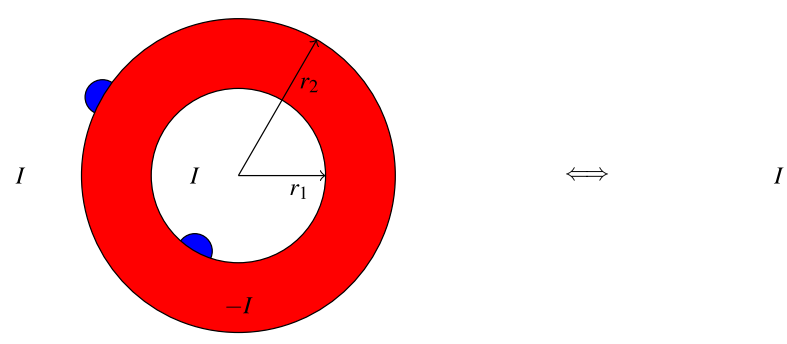

We have, by Lemma 2.1,

$$
\left\|u_{\delta}\right\|_{H^{1}(\Omega)} \leq C \operatorname{Data}(f, \delta),
$$

where

$$
\operatorname{Data}(f, \delta):=\frac{1}{\delta}\left|\Im \int_{\Omega} f \bar{u}_{\delta}\right|+\|f\|_{L^{2}(\Omega)}^{2} .
$$

As in the proof of Theorem 2.1, define $u_{1, \delta} \in H_{\text {loc }}^{1}\left(\mathbb{R}^{2} \backslash B_{r_{2}}\right)$ and $u_{2, \delta} \in H^{1}\left(B_{r_{3}}\right)$ as follows

$$
u_{1, \delta}=u_{\delta} \circ F^{-1} \text { in } \mathbb{R}^{2} \backslash B_{r_{2}} \quad \text { and } \quad u_{2, \delta}=u_{1, \delta} \circ G^{-1} \text { in } B_{r_{3}} .
$$

Set

$$
S=\left(B_{r_{3}} \backslash B_{r_{2}}\right) \cap\left(B\left(x_{2}, r_{0}\right) \cup G \circ F\left(B\left(x_{1}, r_{0}\right) \cap B_{r_{1}}\right)\right) .
$$

By Lemma 2.3, we have

$$
u_{1, \delta}-u_{\delta}=0 \quad \text { and }\left.\quad \partial_{r} u_{1, \delta}\right|_{\text {ext }}-\partial_{r} u_{\delta}=-\left.i \delta \partial_{r} u_{1, \delta}\right|_{\text {ext }} \text { on } \partial B_{r_{2}} \backslash \partial S,
$$

and

$$
u_{2, \delta}-u_{1, \delta}=0 \quad \text { and }\left.\quad \partial_{r} u_{2, \delta}\right|_{\text {int }}-\partial_{r} u_{1, \delta}=\left.i \delta \partial_{r} u_{1, \delta}\right|_{\text {int }} \text { on } \partial B_{r_{3}} \backslash \partial S .
$$

Applying Lemma 2.3, we obtain

$$
\Delta u_{1, \delta}=0 \text { in } B_{r_{3}} \backslash B_{r_{2}}
$$

and

$$
\Delta u_{2, \delta}=0 \text { in } B_{r_{3}} \backslash\left((G \circ F)\left(B\left(x_{1}, r_{0}\right) \cap B_{r_{1}}\right)\right) .
$$

Recall that

$$
\Delta u_{\delta}=0 \text { in }\left(B_{r_{3}} \backslash B_{r_{2}}\right) \backslash \mathcal{C} .
$$

Denote $x_{3} \in \partial B_{r_{3}}$ the image of $x_{1}$ by $F$. The new key ingredient in comparison with the approach used in the proof of Theorem 3.1 is the fact that there exist two constants $R_{2}, R_{3}>0$ such that if $r_{0}$ is small enough and if one defines

$$
O_{2}=B_{r_{2}} \cup\left\{\left|z-x_{2}\right|<R_{2}\right\}, \quad O_{3}=B_{r_{3}} \backslash\left\{\left|z-x_{3}\right|<R_{3}\right\}, \quad \text { and } \quad O=O_{3} \backslash O_{2},
$$

then

$$
\left\|u_{1, \delta}-u_{\delta}\right\|_{H^{1 / 2}\left(\partial O_{2}\right)}+\left\|\partial_{r}\left(u_{1, \delta}-u_{\delta}\right)\right\|_{H^{-1 / 2}\left(\partial O_{2}\right)} \leq C \delta^{\beta}\left\|u_{\delta}\right\|_{H^{1}\left(B_{r_{3}}\right)}
$$

and

$$
\left\|u_{2, \delta}-u_{1, \delta}\right\|_{H^{1 / 2}\left(\partial O_{3}\right)}+\left\|\partial_{r}\left(u_{2, \delta}-u_{1, \delta}\right)\right\|_{H^{-1 / 2}\left(\partial O_{3}\right)} \leq C \delta^{\beta}\left\|u_{\delta}\right\|_{H^{1}\left(B_{r_{3}}\right)} .
$$


The details of the proof of this fact, which are out of the scope of this survey, are given in [28, Section 3.1]. Define

$$
\hat{u}_{\delta}=\left\{\begin{array}{cl}
u_{\delta} & \text { in } \Omega \backslash O_{3}, \\
u_{2, \delta}-\left(u_{1, \delta}-u_{\delta}\right) & \text { in } O \\
u_{2, \delta} & \text { in } O_{2}
\end{array}\right.
$$

Then, $\hat{u}_{\delta} \in H^{1}(\Omega \backslash \partial O)$ with $\hat{u}_{\delta}=0$ on $\partial \Omega$ is a solution of the equation

$$
\Delta \hat{u}_{\delta}=f \text { in } \Omega \backslash \partial O .
$$

This implies, by (4.9) and (4.10),

$$
\left\|\hat{u}_{\delta}-\hat{u}\right\|_{H^{1}(\Omega \backslash \partial O)} \leq C \delta^{\beta}\left\|u_{\delta}\right\|_{H^{1}\left(B_{r_{3}}\right)} .
$$

Since $\beta>1 / 2$, it follows from (4.5) that

$$
\left\|\hat{u}_{\delta}\right\|_{H^{1}(\Omega \backslash \partial O)} \leq C\|f\|_{L^{2}(\Omega)} .
$$

This in turn implies that

$$
\operatorname{Data}(f, \delta) \leq C \delta^{-1}\|f\|_{L^{2}(\Omega)}^{2}
$$

and

$$
\left\|\hat{u}_{\delta}-\hat{u}\right\|_{H^{1}(\Omega \backslash \partial O)} \leq C \delta^{\beta-1 / 2}\|f\|_{L^{2}(\Omega)} .
$$

Involving the arguments used in the last part of the proof of Theorem 3.1, we have, for $n \geq 1$,

$$
\operatorname{Data}(f, \delta) \leq C \delta^{\beta\left(1+\cdots+1 / 2^{n-1}\right)-\left(1+\cdots+1 / 2^{n}\right)-1}\|f\|_{L^{2}(\Omega)}^{2}
$$

and

$$
\left\|\hat{u}_{\delta}-\hat{u}\right\|_{H^{1}(\Omega \backslash \partial O)} \leq C \delta^{\beta\left(1+\cdots+1 / 2^{n}\right)-\left(1 / 2+\cdots+1 / 2^{n+1}\right)}\|f\|_{L^{2}(\Omega)} .
$$

The conclusion follows by taking $n$ sufficiently large.

Remark 4.1 As mentioned, one of the key ingredients are (4.9) and (4.10). This is based on a three-sphere inequality with a partial information; see [24, Section 3.1]. The proof of this result also involves the properties of conformal maps. A variant of these inequalities holds for the Helmholtz equation in two dimensions. Due to the use of the conformal maps in two dimensions, we do not know if the variants of (4.9) and (4.10) hold for three dimensions. Nevertheless, a modification of the cloaking construction can be made to obtain a cloaking device that can cloak some finite region near by. The modification is based on the concept of doubly complementary media that was first introduced in [24] with its roots in [22]. The interested reader can find a detailed discussion in [28].

Invoking ideas similar to those in the proof of Theorem 4.1, we establish, see [28, Proposition 3.2], that

Proposition 4.1 Let $d=2,0<\delta<1,0<r_{0}<r_{1}<r_{2}$, and $x_{3} \in \partial B_{r_{3}}$ with $r_{3}=r_{2}^{2} / r_{1}$. Assume that $B_{r_{3}} \subset \subset \Omega$ and let $f \in L^{2}(\Omega)$ with supp $f \subset \Omega \backslash B_{r_{3}}$. Let $a_{c}$ be a symmetric uniformly elliptic matrix-valued function defined in $B\left(x_{3}, r_{0}\right) \cap B_{r_{3}}$. Let $u_{\delta} \in H_{0}^{1}(\Omega)$ be the unique solution of (3.21) where

$$
\operatorname{div}\left(s_{\delta} A \nabla u_{\delta}\right)=f \text { in } \Omega .
$$


Here $A$ is given by (3.4) where

$$
a_{e}=\left\{\begin{array}{l}
a_{c} \text { in } B\left(x_{3}, r_{0}\right) \cap B_{r_{3}}, \\
I \quad \text { in }\left(B_{r_{3}} \backslash B_{r_{2}}\right) \backslash B\left(x_{3}, r_{0}\right) .
\end{array}\right.
$$

There exists $r_{*}>0$ depending only on $r_{1}$ and $r_{2}$ such that if $r_{0}<r_{*}$, then

$$
u_{\delta} \rightarrow \hat{u} \text { in } L^{2}\left(\Omega \backslash B_{r_{3}}\right) \text {. }
$$

Here $\hat{u} \in H_{0}^{1}(\Omega)$ is the unique outgoing solution to the equation

$$
\operatorname{div}(\hat{A} \nabla \hat{u})=f \text { in } \Omega, \text { where } \hat{A}= \begin{cases}a_{c} & \text { in } B\left(x_{0}, r_{0}\right) \cap B_{r_{3}}, \\ I & \text { otherwise. }\end{cases}
$$

From (4.16) and (4.17), one concludes that the object in $B_{r_{3}} \backslash B_{r_{2}}$ is not cloaked by its complementary medium in $B_{r_{2}} \backslash B_{r_{1}}$ as suggested in [19] and as is usually accepted in the literature.

\section{Electromagnetic Wave Propagation in Media Consisting of Dispersive Metamaterials}

The fundamental Maxwell's equations-without source-are

$$
\left\{\begin{array}{l}
\partial_{t} D(t, x)=\nabla \times H(t, x), \\
\partial_{t} B(t, x)=-\nabla \times E(t, x),
\end{array} \quad \text { for } t \in \mathbb{R}, x \in \mathbb{R}^{3},\right.
$$

where $E \in \mathbb{R}^{3}$ (resp. $H \in \mathbb{R}^{3}$ ) is the electric (resp. magnetic) field and $D \in \mathbb{R}^{3}$ (resp. $B \in \mathbb{R}^{3}$ ) is the electric (resp. magnetic) induction field. In order to close the system (5.1), one adds constitutive relations that express $D$ and $B$ as functions of $E$ and $H$. For dispersive media, these relations are frequency dependent. Taking these constitutive relations into account, the corresponding system of (5.1) in the time domain has the form

$\left\{\begin{array}{l}\epsilon_{r e l}(x) \partial_{t} E(t, x)+\left(\lambda_{e e} * E\right)(t, x)+\left(\lambda_{e m} * H\right)(t, x)=\nabla \times H(t, x), \\ \mu_{r e l}(x) \partial_{t} H(t, x)+\left(\lambda_{m e} * E\right)(t, x)+\left(\lambda_{m m} * H\right)(t, x)=-\nabla \times E(t, x),\end{array} \quad t \in \mathbb{R}, x \in \mathbb{R}^{3}\right.$,

where $*$ stands for the convolution with respect to time $t$. Here the following conventions/assumptions are imposed on $\epsilon_{r e l}, \mu_{r e l}$, and $\lambda_{i j}$ for $i, j \in\{e, m\}$ :

$\epsilon_{\text {rel }}$ and $\mu_{\text {rel }}$ are two $3 \times 3$ real symmetric uniformly elliptic matrices defined in $\mathbb{R}^{3}$.

and

$$
\widehat{\lambda_{i j}}, \lambda_{i j} \in L_{\text {loc }}^{1}\left(\mathbb{R}, L^{\infty}\left(\mathbb{R}^{3}\right)^{3 \times 3}\right), \text { and } \lambda_{i j} \text { is real-valued, } \quad \text { for }(i, j) \in\{e, m\}^{2} .
$$

In this section, for a time-dependent quantity $X(t, x)$, its temporal Fourier transform is given by

$$
\widehat{X}(\omega, x):=\frac{1}{\sqrt{2 \pi}} \int_{\mathbb{R}} X(t, x) e^{i \omega t} d t, \quad \text { for } \omega \in \mathbb{R}, x \in \mathbb{R}^{3} .
$$

Let $\chi_{i j}$ be the susceptibilities that characterizes the dispersive effects of the medium. The connection between $\lambda_{i j}$ and $\chi_{i j}$ is

$$
\widehat{\lambda_{i j}}(\omega, x):=-i \omega \widehat{\chi_{i j}}(\omega, x), \quad \text { for }(i, j) \in\{e, m\}^{2}, \omega \in \mathbb{R}, x \in \mathbb{R}^{3} .
$$


The permittivity $\epsilon$ and the permeability $\mu$ of the medium are given by

$$
\widehat{\epsilon}:=\epsilon_{r e l}+\widehat{\chi}_{e e} \quad \text { and } \widehat{\mu}:=\mu_{r e l}+\widehat{\chi}_{m m} .
$$

The details of deriving (5.2) from (5.1) using the appropriate assumptions on dispersive media are given in [34, Section 2].

Two fundamental assumptions physically relevant to the model, causality and passivity, are imposed.

Causality the effect cannot precede the cause, i.e., the present states of the system depend only on its states in the past. Mathematically, one requires

$$
\lambda_{i j}(t)=0, \quad \text { for all } t<0 \text { and for all }(i, j) \in\{e, m\}^{2} .
$$

Under this assumption, we have, for $(i, j) \in\{e, m\}^{2}$,

$$
\left(\lambda_{i j} * X\right)(t, \cdot)=\int_{-\infty}^{t} \lambda(t-\tau, \cdot) X(\tau, \cdot) d \tau=\int_{0}^{\infty} \lambda(\tau, \cdot) X(t-\tau, \cdot) d \tau, \quad \text { for } t \in \mathbb{R}
$$

Passivity One assumes, for almost every $x \in \mathbb{R}^{3}$, for almost every $\omega \in \mathbb{R}$, and for all $X \in \mathbb{C}^{65}$, that ${ }^{6}$

$$
\operatorname{Re}\left(\left[\begin{array}{cc}
\widehat{\lambda_{e e}}(\omega, x) & \widehat{\lambda_{e m}}(\omega, x) \\
\widehat{\lambda_{m e}}(\omega, x) & \widehat{\lambda_{m m}}(\omega, x)
\end{array}\right] X \cdot \bar{X}\right) \geq 0
$$

Assumption (5.10) means that the medium is dissipative, i.e., it does not produce electromagnetic energy by itself.

In the anisotropic case $\left(\chi_{e m}=\chi_{m e}=0\right)$, condition (5.10) is equivalent to ${ }^{7}$

$$
\omega \operatorname{Im} \widehat{\epsilon}(\omega), \omega \operatorname{Im} \widehat{\mu}(\omega) \geq 0, \quad \text { for almost all } \omega \in \mathbb{R} .
$$

Condition (5.11) ensures that when small loss is added, the problem associated with the outgoing (Silver-Müller) condition at infinity is well-posed (see, e.g., [29]). Adding a small loss is the standard mechanism to study phenomena related to metamaterials in the frequency domain. Nevertheless, condition (5.11) does not exclude the ill-posedness in the frequency domain when the loss is 0 (see [26, Proposition 2]). As one sees later, even if the problem is ill-posed in the frequency domain for some frequency, the well-posedness is roughly ensured for the problem in the time domain under the causality and passivity conditions mentioned above (see Theorem 5.1).

One of typical classes of dispersive anisotropic media $\left(\chi_{m e}=\chi_{e m}=0\right)$ satisfying the condition (5.4), the causality (5.8), and the passivity (5.10) is the class of media obeying Lorentz' model. For a homogeneous isotropic medium, the susceptibilities $\chi_{e e}$ and $\chi_{m m}$ are of the form (see, e.g., [15, (7.51)])

$$
\widehat{\chi}(\omega)=\sum_{\ell=1}^{n} \frac{\omega_{p, \ell}^{2}}{\omega_{0, \ell}^{2}-\omega^{2}-2 i \gamma_{\ell} \omega} I, \quad \text { for } \omega \in \mathbb{R},
$$

\footnotetext{
${ }^{5}$ Here $\mathbb{C}$ denotes the set of complex numbers.

${ }^{6}$ Here $\cdot$ stands for the Euclidean scalar product in $\mathbb{C}^{6}$.

${ }^{7}$ Here for a $3 \times 3$ matrix $A$, we denote $A \leq 0$ if $A x \cdot x \leq 0$ for all $x \in \mathbb{R}^{3}$.
} 
where $\omega_{p, \ell}$ (resp. $\omega_{0, \ell}$ and $\gamma_{\ell}$ ) are positive (resp. non-negative) material constants (recall that $I$ is the identity matrix). Using the residue theorem, one can show (see, e.g., [15, (7.110)]) that for $t \in \mathbb{R}$ one has

$$
\begin{aligned}
& \chi(t)=\sqrt{2 \pi} \vartheta(t) \sum_{\ell=1}^{n} \omega_{p, \ell}^{2} \frac{\sin \left(\nu_{\ell} t\right)}{v_{\ell}} e^{-\gamma_{\ell} t} I_{3} \text { and } \\
& \lambda(t)=\sqrt{2 \pi} \vartheta(t) \sum_{\ell=1}^{n} \omega_{p, \ell}^{2} \frac{d}{d t}\left(\frac{\sin \left(\nu_{\ell} t\right)}{\nu_{\ell}} e^{-\gamma_{\ell} t}\right) I_{3},
\end{aligned}
$$

where $v_{\ell}^{2}=\omega_{0, \ell}^{2}-\gamma_{\ell}^{2}$ (if $\omega_{0, \ell}>\gamma_{\ell}$ ) and $\vartheta$ is the Heaviside function, i.e., $\vartheta(t)=1$ if $t \geq 0$ and $\vartheta(t)=0$ otherwise. Here $\lambda$ is defined in such a way that $\widehat{\lambda}(\omega)=-i \omega \widehat{\chi}(\omega)$ for $\omega \in \mathbb{R}$.

We study (5.2) under the form of the initial problem at the time $t=0$, assuming that the data are known in the past $t<0$. Set

$$
\left(\lambda_{i j} \star X\right)(t, \cdot):=\int_{0}^{t} \lambda(t-\tau, \cdot) X(\tau, \cdot) d \tau, \quad \text { for } t>0 .
$$

For $X=E$ or $H$, under the causality assumption (5.8)-(5.9), one has for $t>0$ that

$$
\begin{aligned}
\left(\lambda_{i j} * X\right)(t, \cdot) & =\int_{0}^{t} \lambda_{i j}(t-\tau, \cdot) X(\tau, \cdot) d \tau+\int_{-\infty}^{0} \lambda_{i j}(t-\tau, \cdot) X(\tau, \cdot) d \tau \\
& =\left(\lambda_{i j} \star X\right)(t, \cdot)+\int_{-\infty}^{0} \lambda_{i j}(t-\tau, \cdot) X(\tau, \cdot) d \tau .
\end{aligned}
$$

Hence, if the data are known for the past $t<0$, then the last term is known at time $t>0$. With the presence of sources, one can then reformulate system (5.2) under the form

$$
\left\{\begin{array}{l}
\epsilon_{r e l}(x) \partial_{t} E(t, x)+\left(\lambda_{e e} \star E\right)(t, x)+\left(\lambda_{e m} \star H\right)(t, x)=\nabla \times H(t, x)+f_{e}(t, x), \\
\mu_{r e l}(x) \partial_{t} H(t, x)+\left(\lambda_{m e} \star E\right)(t, x)+\left(\lambda_{m m} \star H\right)(t, x)=-\nabla \times E(t, x)+f_{m}(t, x), \\
E(0, x)=E_{0}(x), H(0, x)=H_{0}(x),
\end{array}\right.
$$

for $t>0$ and $x \in \mathbb{R}^{3}$. Here $E_{0}$ and $H_{0}$ are the initial data at time $t=0$, and $f_{e}, f_{m}$ are given fields that can be considered as "effective" sources since they also take into account the last terms in (5.15).

Set

$$
\begin{gathered}
u:=\left[\begin{array}{l}
E \\
H
\end{array}\right], \quad u_{0}:=\left[\begin{array}{l}
E_{0} \\
H_{0}
\end{array}\right], \quad f:=\left[\begin{array}{l}
f_{e} \\
f_{m}
\end{array}\right], \quad \mathbb{A} u:=\left[\begin{array}{l}
\nabla \times H \\
-\nabla \times E
\end{array}\right], \\
\Lambda:=\left[\begin{array}{ll}
\lambda_{e e} & \lambda_{e m} \\
\lambda_{m e} & \lambda_{m m}
\end{array}\right] \quad \text { and } \quad M:=\left[\begin{array}{ll}
\epsilon_{\text {rel }} & 0 \\
0 & \mu_{\text {rel }}
\end{array}\right] .
\end{gathered}
$$

System (5.16) can then be rewritten in the following compact form:

$$
\left\{\begin{array}{l}
M(x) \partial_{t} u(t, x)+(\Lambda \star u)(t, x)=\mathbb{A} u(t, x)+f(t, x), \quad \text { for } t>0, x \in \mathbb{R}^{3} . \\
u(0, x)=u_{0}(x),
\end{array}\right.
$$

Define

$$
\mathcal{H}:=L^{2}\left(\mathbb{R}^{3}\right)^{3} \times L^{2}\left(\mathbb{R}^{3}\right)^{3} \quad \text { and } \quad \mathcal{V}:=H_{\text {curl }}\left(\mathbb{R}^{3}\right) \times H_{\text {curl }}\left(\mathbb{R}^{3}\right),
$$

equipped with the standard inner products induced from $L^{2}\left(\mathbb{R}^{3}\right)^{3}$ and $H_{\text {curl }}\left(\mathbb{R}^{3}\right)$. One can verify that $\mathcal{H}$ and $\mathcal{V}$ are Hilbert spaces.

We also denote

$\mathcal{M}_{6}\left(L^{\infty}\left(\mathbb{R}^{3}\right)\right)$ as the space of $6 \times 6$ real matrices whose entries are $L^{\infty}\left(\mathbb{R}^{3}\right)$ functions. 
In what follows, in the time domain, we only consider real quantities.

Concerning the well-posedness of (5.19), we prove, see [34, Theorem 3.1],

Theorem 5.1 Let $T \in(0,+\infty), u_{0} \in \mathcal{H}, f \in L^{1}(0, T ; \mathcal{H})$, and $\Lambda \in L^{1}(0, T$; $\mathcal{M}_{6}\left(L^{\infty}\left(\mathbb{R}^{3}\right)\right)$. Assume that (5.3), (5.4), (5.8) and (5.10) hold. There exists a unique weak solution $u \in L^{\infty}(0, T ; \mathcal{H})$ of $(5.19)$ on $(0, T)$. Moreover, the following estimate holds

$$
\langle M u(t, \cdot), u(t, \cdot)\rangle_{\mathcal{H}} \leq\left(\left\langle M u_{0}, u_{0}\right\rangle_{\mathcal{H}}^{1 / 2}+C \int_{0}^{t}\|f(s, \cdot)\|_{\mathcal{H}} d s\right)^{2} \quad \text { in }(0, T),
$$

where $C$ is a positive constant depending only on the coercivity of $M$.

The notion of weak solutions for (5.19) is

Definition 5.1 Let $T \in(0,+\infty), u_{0} \in \mathcal{H}$ and $f \in L^{1}(0, T ; \mathcal{H})$. A function $u \in$ $L^{\infty}(0, T ; \mathcal{H})$ is called a weak solution of $(5.19)$ on $[0, T]$ if

$\frac{d}{d t}\langle M u(t, \cdot), v\rangle_{\mathcal{H}}+\langle(\Lambda \star u)(t, \cdot), v\rangle_{\mathcal{H}}=\langle u(t, \cdot), \mathbb{A} v\rangle_{\mathcal{H}}+\langle f(t, \cdot), v\rangle_{\mathcal{H}}$ in $(0, T)$ for all $v \in \mathcal{V}$,

and

$$
u(0, \cdot)=u_{0}
$$

Remark 5.1 One can easily check that if $u$ is a smooth solution and decays sufficiently at infinity, then $u$ is a weak solution by integration by parts, and that if $u$ is a weak solution and smooth, then $u$ is a classical solution.

We next discuss the finite speed propagation for (5.19). In what follows, $B(a, R)$ stands for the ball in $\mathbb{R}^{3}$ of radius $R>0$ and centered at $a \in \mathbb{R}^{3}$. Set

$$
c(x):=\gamma_{e}(x) \gamma_{m}(x), \quad \text { for } x \in \mathbb{R}^{3},
$$

where $\gamma_{e}(x)$ and $\gamma_{m}(x)$ are the largest eigenvalues of $\epsilon_{\text {rel }}(x)^{-1 / 2}$ and $\mu_{\text {rel }}(x)^{-1 / 2}$, respectively. According to assumption (5.3), $c(x)$ is bounded above and below by a positive constant. For $a \in \mathbb{R}^{3}$ and $R>0$, we denote

$$
c_{a, R}:=\operatorname{ess~sup}_{x \in B(a, R)} c(x) .
$$

The following result is on the finite speed propagation of (5.19); see [34, Theorem 3.2]:

Theorem 5.2 Let $R>0, a \in \mathbb{R}^{3}$, and $u_{0} \in \mathcal{H}$. For $T>R / c_{a, R}$, let $f \in L^{1}(0, T ; \mathcal{H})$ and $\Lambda \in L^{1}\left(0, T ; \mathcal{M}_{6}\left(L^{\infty}\left(\mathbb{R}^{3}\right)\right)\right.$. Assume that (5.3), (5.4), (5.8), and (5.10) hold,

$$
\operatorname{supp} u_{0} \cap B(a, R)=\emptyset \text {, }
$$

and

$$
\operatorname{supp} f(t, \cdot) \cap B\left(a, R-c_{a, R} t\right)=\emptyset, \quad \text { for almost every } t \in\left(0, R / c_{a, R}\right) .
$$

Let $u \in L^{\infty}(0, T ; \mathcal{H})$ be the unique weak solution of $(5.19)$ on $(0, T)$. Then

$$
\operatorname{supp} u(t, \cdot) \cap B\left(a, R-c_{a, R} t\right)=\emptyset, \quad \text { for almost every } t \in\left(0, R / c_{a, R}\right) .
$$


We briefly mention here the ideas of the proofs of Theorems 5.1 and 5.2. The construct of a solution in Theorem 5.1 is based on the Galerkin method. One of the key observations is the following inequality

$$
\int_{0}^{t}\langle(\Lambda \star v)(s, \cdot), v(s, \cdot)\rangle_{\mathcal{H}} d s \geq 0, \quad \text { for } v \in L^{\infty}(0, T ; \mathcal{H}), t \in(0, T) .
$$

Similar observations in the acoustic setting were used in different contexts; see, e.g., [35, 36]. The inequality (5.30) plays an important role in deriving the following estimate for an approximate solution $u_{n}$ after multiplying the equation of $u_{n}$ by $u_{n}$ and integrating by parts, which gives

$$
\left\langle M u_{n}(t, \cdot), u_{n}(t, \cdot)\right\rangle_{\mathcal{H}} \leq\left\langle M u_{n}(t=0, \cdot), u_{n}(t=0, \cdot)\right\rangle_{\mathcal{H}}+2 \int_{0}^{t}\|f(s, \cdot)\|_{\mathcal{H}}\left\|u_{n}(s, \cdot)\right\|_{\mathcal{H}} d s .
$$

By Gronwall's lemma, this in turn implies the desired estimate for a solution $u$ obtained via the standard compactness argument; see, e.g., [12]. The uniqueness of $u$ is quite standard as in the standard wave equations after noting (5.30). The proof of Theorem 5.2 is standard via (5.30) if one knows that the solution $u$ is regular. To overcome the lack of the regularity of $u$, we consider the function

$$
U(t, x):=\int_{0}^{t} u(s, x) d s, \text { for } t \in[0, T), x \in \mathbb{R}^{3}
$$

and show that

$$
\operatorname{supp} U(t, \cdot) \cap B\left(a, R-c_{a, R} t\right)=\emptyset, \quad \text { for almost every } t \in\left(0, R / c_{a, R}\right) .
$$

This yields the desired conclusion. As far as we know, the proof of finite speed propagation for energy solutions is not presented in standard references on partial differential equations.

\section{Other Topics and Future Directions}

Some interesting aspects of NIMs are not discussed in this survey, such as the stability of NIMs and cloaking a source via anomalous localized resonance, because we have nothing new to add to these topics. The stability of NIMs in the frequency domain for acoustic waves was investigated by Costabel and Stephan in 1985 [11] using the integral method. Later, this problem was studied by the integral method and the pseudo-differential operators theory [38] and by the $T$-coercivity approach (see [5,6] and the references therein). In these works, the well-posedness was established in the Fredholm sense in $H^{1}$, meaning that the compactness holds; the existence and the uniqueness are not discussed. Recently [26], we introduced a new approach to study the stability aspect of NIMs. More precisely, we investigated the well-posedness of the Helmholtz equations involving sign-changing coefficients. Our approach involved the study of Cauchy problems, which are derived by reflections in the spirit of the proofs presented in Sections 2, 3, and 4 using the change of variables formula in Lemma 2.3. We then proposed various methods to study these Cauchy problems. One method was via the prominent work of Agmon, Douglis, and Nirenberg [1] (via Fourier analysis or fundamental solutions) and others were based on variational methods/multiplier techniques. In consequence, we can unify and extend largely known works. In particular, we proved that (see [26, Corollary 1]) the well-posedness holds if, under some smoothness assumptions,

$$
A_{+}>A_{-} \text {on } \Gamma \text { or } A_{+}<A_{-} \text {on } \Gamma,
$$


for all connected component $\Gamma$ of the sign-changing coefficient interface, $A_{+}$is the restriction of $A$ in the region $A>0$, and $A_{-}$is the restriction of $-A$ in the region $A<0$. We also showed that the complementary property of media is almost necessary for the occurrence of resonance (see [26, Proposition 2]). A numerical algorithm in the spirit of this approach was also studied in [2].

The second aspect we do not discuss in this survey is cloaking a source via anomalous localized resonance. This cloaking technique is relative due to the fact that the power, which is roughly speaking the standard energy of the fields in the region of NIMs multiplied by the loss, must be normalized for the cloaking purpose. This phenomenon was observed by Milton and Nicorovici in [21] (see also [20]) for a symmetrical radial structures in a twodimensional quasistatic regime and was considered in a general setting, the setting of doubly complementary media in [24, 30] for the acoustic regime (see also [3, 9, 17] for related results in some specific settings). It has been shown [24, 30] that (i) cloaking a source via anomalous localized resonance appears if and only if the power blows up; (ii) the power blows up if the source is located "near" the plasmonic layer made of NIMs; and (iii) the power remains bounded if the source is far away from the plasmonic layer. It is worth noting that there is no connection between the blow up of the power and the localized resonance in general [32]. Finally, we want to mention that the design of metamaterials poses new and interesting problems that are being extensively investigated in the litterature; see $[8,10,14$, $18]$ and the references therein.

An interesting direction concerning NIMs or more generally metamaterials is to study these metamaterials in the time domain. For example, it would be interesting to understand conditions under which the energy of solutions of the Maxwell equations considered in Section 5 decays in any bounded domain; this is known for (standard) positive index media. Another interesting question would be to investigate the limiting amplitude principle, which concerns the behavior of the fields in the time domain generated by a harmonic forcing term for large time. In some particular settings, the limiting amplitude principle was already considered in $[13,16]$, but the question for a general setting remains open.

Acknowledgements This paper is an extended version of the lecture given by the author at VIASM annual meeting in 2017 at Vietnam Institute for Advanced Study in Mathematics. The author warmly thanks the institute for the hospitality.

\section{References}

1. Agmon, S., Douglis, A., Nirenberg, L.: Estimates near the boundary for solutions of elliptic partial differential equations satisfying general boundary conditions. II. Comm. Pure Appl. Math. 17, 35-92 (1964)

2. Abdulle, A., Huber, M.E., Lemaire, S.: An optimization-based numerical method for diffusion problems with sign-changing coefficients. C. R. Math. Acad. Sci. Paris 355, 472-478 (2017)

3. Ammari, H., Ciraolo, G., Kang, H., Lee, H., Milton, G.W.: Anomalous localized resonance using a folded geometry in three dimensions. Proc. R. Soc. Lond. Ser. A 469, 20130048 (2013)

4. Bethuel, F., Brezis, H., Helein, F.: Ginzburg Landau Vortices. Progress in Nonlinear Differential Equations and Their Applications, vol. 13. Birkhäuser, Boston (1994)

5. Bonnet-Ben Dhia, A.S., Chesnel, L., Ciarlet, P.: T-coercivity for scalar interface problems between dielectrics and metamaterials. ESAIM Math. Model. Numer. Anal. 46, 1363-1387 (2012)

6. Bonnet-Ben Dhia, A.S., Ciarlet, P., Zwölf, C.M.: A new compactness result for electromagnetic waves. Application to the transmission problem between dielectrics and metamaterials. Math. Models Methods Appl. Sci. 18, 1605-1631 (2008)

7. Bonnetier, E., Nguyen, H.-M.: Superlensing using hyperbolic metamaterials: the scalar case. J. Éc. polytech. Math. 4, 973-1003 (2017) 
8. Bouchitté, G., Felbacq, D.: Homogenization near resonances and artificial magnetism from dielectrics. C. R. Math. Acad. Sci. Paris 339, 377-382 (2004)

9. Bouchitté, G., Schweizer, B.: Cloaking of small objects by anomalous localized resonance. Quart. J. Mech. Appl. Math. 63, 437-463 (2010)

10. Chen, Y., Lipton, R.: Resonance and double negative behavior in metamaterials. Arch. Ration. Mech. Anal. 209, 835-868 (2013)

11. Costabel, M., Stephan, E.: A direct boundary integral equation method for transmission problems. J. Math. Anal. Appl. 106, 367-413 (1985)

12. Evans, L.C.: Partial Differential Equations Graduate Studies in Mathematics, vol. 19. American Mathematical Society, Providence (1998)

13. Gralak, B., Tip, A.: Macroscopic Maxwell's equations and negative index materials. J. Math. Phys. 51, 052902 (2010)

14. Guenneau, S., Zolla, F.: Homogenization of 3D finite chiral photonic crystals. Phys. B: Condens. Matter 394, 145-147 (2007)

15. Jackson, J.D.: Classical Electrodynamics. Wiley, NY (1999)

16. Cassier, M., Hazard, C., Joly, P.: Spectral theory for Maxwell's equations at the interface of a metamaterial. Part I: generalized Fourier transform. Comm. Partial Diff. Equat. 42(11), 1707-1748 (2017)

17. Kohn, R.V., Lu, J., Schweizer, B., Weinstein, M.I.: A variational perspective on cloaking by anomalous localized resonance. Comm. Math. Phys. 328, 1-27 (2014)

18. Kohn, R.V., Shipman, S.P.: Magnetism and homogenization of microresonators. Multiscale Model Simul. 7, 62-92 (2008)

19. Lai, Y., Chen, H., Zhang, Z.Q., Chan, C.T.: Complementary media invisibility cloak that cloaks objects at a distance outside the cloaking shell. Phys. Rev. Lett. 102, 093901 (2009)

20. Milton, G.W., Nicorovici, N.A., McPhedran, R.C., Podolskiy, V.A.: A proof of superlensing in the quasistatic regime, and limitations of superlenses in this regime due to anomalous localized resonance. Proc. R. Soc. Lond. Ser. A 461, 3999-4034 (2005)

21. Milton, G.W., Nicorovici, N.A.P.: On the cloaking effects associated with anomalous localized resonance. Proc. R. Soc. Lond. Ser. A 462, 3027-3059 (2006)

22. Nguyen, H.-M.: Asymptotic behavior of solutions to the Helmholtz equations with sign changing coefficients. Trans. Am. Math. Soc. 367, 6581-6595 (2015)

23. Nguyen, H.-M.: Superlensing using complementary media. Ann. Inst. H. Poincaré Anal. Non Linéaire 32, 471-484 (2015)

24. Nguyen, H.-M.: Cloaking via anomalous localized resonance for doubly complementary media in the quasistatic regime. J. Eur. Math. Soc. (JEMS) 17, 1327-1365 (2015)

25. Nguyen, H.-M.: Cloaking using complementary media in the quasistatic regime. Ann. Inst. H. Poincaré Anal. Non Linéaire 33, 1509-1518 (2016)

26. Nguyen, H.-M.: Limiting absorption principle and well-posedness for the Helmholtz equation with sign changing coefficients. J. Math. Pures Appl. 106, 342-374 (2016)

27. Nguyen, H.-M.: Negative index materials and their applications: recent mathematics progress. Chin. Ann. Math. Ser. B 38, 601-628 (2017)

28. Nguyen, H.-M.: Cloaking an arbitrary object via anomalous localized resonance: the cloak is independent of the object. SIAM J. Math. Anal. 49, 3208-3232 (2017)

29. Nguyen, H.-M.: Superlensing using complementary media and reflecting complementary media for electromagnetic waves. Adv. Nonlinear Anal. to appear, https://doi.org/10.1515/anona-2017-0146

30. Nguyen, H.-M.: Cloaking via anomalous localized resonance for doubly complementary media in the finite frequency regime. J. Anal. Math. to appear, arXiv:1511.08053

31. Nguyen, H.-M.: Cloaking using complementary media for electromagnetic waves. ESAIM Control Optim. Calc. Var., to appear, https://doi.org/10.1051/cocv/2017078

32. Nguyen, H.-M., Nguyen, H.L.: Complete resonance and localized resonance in plasmonic structures. ESAIM: Math. Model. Numer. Anal. 49, 741-754 (2015)

33. Nguyen, H.-M., Nguyen, H.L.: Cloaking using complementary media for the Helmholtz equation and a three spheres inequality for second order elliptic equations. Trans. Am. Math. Soc. Ser. B 2, 93-112 (2015)

34. Nguyen, H.-M., Vinoles, V.: Electromagnetic wave propagation in dispersive metamaterials. submitted, arXiv: 1710.08648

35. Nguyen, H.-M., Nguyen, L.: Generalized impedance boundary conditions for scattering by strongly absorbing obstacles for the full wave equation: the scalar case. Math. Models Methods Appl. Sci. 25, 1927-1960 (2015) 
36. Nguyen, H.-M., Vogelius, M.S.: Approximate cloaking for the full wave equation via change of variables: the Drude-Lorentz model. J. Math. Pures Appl. 106, 797-836 (2016)

37. Nicorovici, N.A., McPhedran, R.C., Milton, G.M.: Optical and dielectric properties of partially resonant composites. Phys. Rev. B 49, 8479-8482 (1994)

38. Ola, P.: Remarks on a transmission problem. J. Math. Anal. Appl. 16, 639-658 (1995)

39. Pendry, J.B.: Negative refraction makes a perfect lens. Phys. Rev. Lett. 85, 3966-3969 (2000)

40. Pendry, J.B.: Perfect cylindrical lenses. Opt. Express 1, 755-760 (2003)

41. Protter, M.H.: Unique continuation for elliptic equations. Trans. Am. Math. Soc. 95, 81-91 (1960)

42. Ramakrishna, S.A., Pendry, J.B.: Focusing light using negative refraction. J. Phys. Condens. Matter 15 , 6345-6364 (2003)

43. Ramakrishna, S.A., Pendry, J.B.: Spherical perfect lens: solutions of Maxwell's equations for spherical geometry. Phys. Rev. B 69, 115115 (2004)

44. Shelby, R.A., Smith, D.R., Schultz, S.: Experimental Verification of a Negative Index of Refraction. Science 292, 77-79 (2001)

45. Veselago, V.G.: The electrodynamics of substances with simultaneously negative values of $\epsilon$ and $\mu$. Usp. Fiz. Nauk 92, 517-526 (1964) 